\title{
Les migrations internationales dans les sources administratives et documentaires turques (1961-2000)
}

Stéphane de Tapia

\section{CpenEdition}

Journals

Édition électronique

URL : https://journals.openedition.org/remi/2634

DOI : $10.4000 /$ remi.2634

ISSN : $1777-5418$

Éditeur

Université de Poitiers

\section{Édition imprimée}

Date de publication : 1 mars 2002

Pagination : 159-198

ISBN : 2-911627-30-X

ISSN : 0765-0752

Référence électronique

Stéphane de Tapia, "Les migrations internationales dans les sources administratives et documentaires turques (1961-2000)», Revue européenne des migrations internationales [En ligne], vol. 18 - $n^{\circ} 1$ | 2002, mis en ligne le 09 juin 2006, consulté le 15 avril 2022. URL : http:// journals.openedition.org/remi/2634 ; DOI : https://doi.org/10.4000/remi.2634

Ce document a été généré automatiquement le 15 avril 2022

(C) Université de Poitiers 


\title{
Les migrations internationales dans les sources administratives et documentaires turques (1961-2000) ${ }^{\text {[id }}$
}

\author{
Stéphane de Tapia
}

Le champ migratoire turc à la fin de 2000

1 La définition, l'historique, les éléments de la construction du champ migratoire turc, ont fait l'objet de nombreuses publications, tant en Turquie qu'en Allemagne, où les références se chiffrent par milliers (Abadan-Unat et Kemiksiz 1986, Boos-Nünning 1990, Schwarz et Ersöz 1990) que dans les autres pays participant de ce champ, France, PaysBas, Belgique, pays scandinaves, voire Australie... Un travail en cours, appuyé sur les références enregistrées à partir de l'ensemble des données transférées par divers correspondants ou disponibles sur publications, comme la base REMISIS, également sur les références consultables sur Internet, permet de rassembler au moins 800 titres en langue française, sans compter plusieurs centaines de petits articles parus dans différents bulletins ou nombre d'articles de presse (titres français, belges, suisses, parfois canadiens, ou versions françaises de textes produits par des organismes internationaux). Encore n'est-ce là qu'une estimation sans doute loin de l'exhaustivité ! (pour une première version provisoire, de Tapia et Dubus 1995) ${ }^{1}$.

2 Nous esquisserons cependant à grands traits ce qu'est le champ migratoire turc à la fin de 2001, soit quarante-quatre années après le déclenchement de cette nouvelle migration internationale. Le dernier rapport disponible du ministère turc du Travail et de la Sécurité Sociale, concernant l'année 1999, a été publié en 2000 (YIHGM 2000). On y apprend qu'officiellement la population turque vivant en émigration atteint 3571771 personnes, dont 3170466 en Europe occidentale - correspondant à peu près à l'Union Européenne, plus la Suisse -, 122880 (135 980 en 1997) au Moyen-Orient et Afrique du Nord (Libye), où les effectifs sont en baisse importante, 50876 en Australie, 55050 dans les pays composant la Communauté des États Indépendants (CEI), 165000 en Amérique du Nord (USA et Canada), 1729 au Japon (apparu dans la liste en 1997). Le nombre de travailleurs enregistrés (avec ou sans chômeurs selon les pays concernés) n'est que de 
1180420 ; il s'agit donc bien maintenant d'une véritable migration de peuplement, mais où la part des travailleurs est, selon les cas, extrêmement variable. Ainsi si la migration est familiale en Europe, elle reste migration de chantier presque exclusivement masculine au Moyen-Orient, en Russie ou en Asie Centrale où cependant la situation semble évoluer très vite. Les chiffres les plus récents, disponibles sur le site Internet du ministère (http://www.calisma.gov.tr), semblent indiquer pour la première fois un tassement de la présence turque à l'étranger. En 1995, Mete Törüner, Directeur Général d'IIBK (Is ve Isçi Bulma Kurumu, Office de Placement et de Recherche d'Emploi), note que chaque année le nombre de personnes placées en Turquie et de migrants placés à l'étranger oscille autour de 250-300 000 et 55000 respectivement (IIBK 1996 : préface). Ces chiffres sur l'emploi, en Turquie comme à l'étranger, sont souvent sousévalués; chacun sait en Turquie que les administrations compétentes sur ces sujets ne contrôlent qu'une faible part des flux, en fait parce qu'il n'existe pas d'allocation chômage jusqu'à la récente réforme de 2000 et que les réseaux informels prévalent largement dans la recherche d'emploi. Ainsi le nombre de demandeurs d'emploi recensés par IIBK - ou l'établissement qui en est issu: Türkiye Is Kurumu (Office du Travail de Turquie) - oscille quant à lui pour la dernière décennie de 335600 en 1995 à plus de 700 000, le chiffre le plus récent en notre possession étant pour 2000 de 768386 (Iskur 2001, sur http://www.calisma.gov.tr ou directement http://www.iskur.gov.tr).

3 La géographie du champ migratoire, au-delà des grandes classifications continentales, présente des évolutions complexes et des stratifications diversifiées d'un aire économique à l'autre. L'épine dorsale du champ reste la Vallée du Rhin lato sensu et, en tous cas, la mégalopole européenne étendue de Londres à Milan, englobant les régions industrielles du Rhin inférieur et moyen, Moselle, Meuse, Main, Neckar et évidemment Ruhr. Les grandes agglomérations que sont Paris et l'Ile de France, Berlin, Munich, Vienne, ... rassemblent généralement plusieurs dizaines de milliers de ressortissants turcs. Stockholm, Copenhague, Londres ou Bruxelles participent de la même logique. Avec la disparition du Rideau de Fer et du Mur de Berlin, l'Allemagne orientale, la Roumanie, la Bulgarie, la Macédoine... sont entrées dans les zones géographiques intéressant les migrants. Les pays de la CEI, Russie, Ukraine, Géorgie d'abord, puis très rapidement républiques turcophones d'Asie Centrale ont attiré, après la perestroika, les entrepreneurs turcs du bâtiment et des travaux publics déjà implantés dans les pays arabes producteurs de pétrole (Arabie Saoudite, Irak, Koweït, Émirats, Libye). Après cette période pourtant récente (1988-1991), d'autres destinations pour l'instant moins stables apparaissent : Israël, Liban, Malaisie, Pakistan, Japon... En Océanie, l'Australie est une cible déjà ancienne, où les caractéristiques de l'immigration se rapprochent très fortement du cas européen - mais où la Nouvelle Zélande semble être une extension récente - tandis qu'en Amérique, on relève les cas différenciés des Etats-Unis (migrations de hauts niveaux de qualification, mais pas uniquement) ${ }^{2}$, du Canada (proche des cas européens et australiens), d'Aruba (avec un grand chantier néerlandais de travaux publics). Nous avons à plusieurs reprises évoqué les étapes de la construction de ce vaste champ migratoire (de Tapia 1992, 1995, 1996, 2000).

Trois phénomènes complémentaires jouent en faveur de l'extension du champ :

5 - la transformation de pays de transit en pays d'immigration (Bulgarie et pays balkaniques, Italie),

6 - la recherche de nouveaux débouchés (CEI et Asie centrale, Malaisie, Pakistan...), 
7 - l'extension locale de régions d'immigration (France du sud et de l'ouest, Allemagne orientale, Liechtenstein, Finlande, Norvège, Nouvelle Zélande...).

8 Sans entrer dans le détail, signalons simplement les destinations citées par le rapport YIHGM pour 1999, d'après les données du ministère de tutelle (IIBK, pour le ministère du Travail et de la Sécurité Sociale) et du ministère des Affaires Étrangères : Allemagne, Arabie Saoudite, Australie, Autriche, Azerbaïdjan, Belgique, Bermudes, Biélorussie, Canada, Chypre-Nord, Croatie, Danemark, Etats-Unis, Éthiopie, Finlande, France, Géorgie, Grande-Bretagne, Guernesey, Israël, Italie, Japon, Jordanie, Kazakhstan, Kirghizie, Libye, Malte, Moldavie, Ouzbékistan, Pays-Bas, Russie, Suède, Suisse, Turkménistan, Ukraine (YIHGM 2000 : 4). Des rapports antérieurs ont cité Afghanistan, Aruba, Bahamas, Égypte, Émirats, Espagne, Iran, Irlande, Koweït, Liban, Malaisie, Norvège, Oman, Pakistan, Roumanie, Tadjikistan, Yémen ; c'est dire la complexité et la diversité des situations qui rythment la vie de cette émigration qui ne date pourtant dans sa forme actuelle - migration internationale de travail - que de 1957 (Abadan $1965)^{3}$.

9 Notre objectif ici n'est pas de refaire l'historique de cette émigration, mais de présenter aux lecteurs francophones les sources turques de l'étude de ce courant migratoire. Nous avons cité sans précision YIHGM et IIBK ; il s'agit de les présenter et d'en faire un bilan critique.

Sources statistiques et flux migratoires : thèmes de recherche

10 La République de Turquie (Türkiye Cumhuriyeti) dispose depuis la fin des années vingt d'un appareil statistique étoffé avec la mise en place d'un Institut National des Statistiques (Devlet Istatistik Enstitüsü) créé selon des modèles français (INSEE et établissements précédents). Il ne faut cependant pas sous-estimer la pratique ottomane des dénombrements qui donnèrent des séries impressionnantes de chiffres que les historiens n'ont pas fini de traiter (Salnameler). La version contemporaine du salname, l'annuaire départemental (yillik) est édité par les préfectures à l'occasion de quelque commémoration marquante, comme la série de 1981 pour le centenaire de la naissance d'Atatürk, série qui a couvert tous les départements. D'autres séries ont été éditées en 1967 ou 1973 et des publications du même type sont souvent relevées pour des groupes qui pensent avoir un message collectif à faire passer: chambres de commerce et d'industries, associations professionnelles, associations de migrants internes ${ }^{4} \ldots$ La pratique de l'archivage des données produites par l'État, dans un régime aussi policé que l'empire ottoman touchait de très nombreux domaines de la vie économique ou juridique. L'administration républicaine a naturellement suivi l'exemple. C'est ainsi que chaque ministère dispose en réalité de ses propres statistiques, reversées ou non, selon le cas, au fonds commun de DIE.

11 Le secteur privé n'est pas en reste, d'une part parce qu'il se doit de fournir des comptes, au propre comme au figuré, à l'Administration, d'autre part parce que cela fait partie de la culture turque. Il est inimaginable qu'un organisme voulant montrer son sérieux ne possède pas au moins un embryon de service statistique lui permettant de contrôler ses propres activités. Ainsi, si les autocaristes doivent chaque année fournir un rapport d'activité au ministère des Transports, l'on pourra trouver des statistiques parfois très évoluées dans les Chambres de Commerce et d'Industrie, auprès des Unions de producteurs, des syndicats professionnels, mais aussi des syndicats ouvriers. Il se peut que ces statistiques soient mal tenues, qu'elles soient en partie occultées, difficiles d'accès, elles n'en sont pas moins existantes; au chercheur de négocier avec des 
partenaires parfois difficiles, mais dignes d'intérêt parce que descendants directs de l'un des empires les plus friands d'archivage au monde! La Turquie est à ce titre l'un des paradis des historiens. Le rapport à l'écrit administratif des citoyens turcs transparaît parfois dans l'émigration, alors que l'on s'attendrait à une culture d'origine plutôt orale parce que très rurale encore dans les années soixante à quatre-vingt, au plus fort de l'émigration ${ }^{5}$. C'est méconnaître le fonctionnement de l'administration turque, même en zone rurale.

12 Pour ce qui nous intéresse ici, il convient de passer en revue plus systématiquement certaines de ces sources statistiques ou documentaires. Elles seront examinées en deux temps: par thèmes de recherche, puis par regroupements institutionnels et tutelles administratives.

Émigration de Turquie

L'émigration proprement dite est du ressort de l'office du Travail et du Placement (IIBK - Is ve Isçi Bulma Kurumu), dont les compétences associent celles de l'ANPE et de l'OMI dans le cas français. Mais elle intéresse aussi le ministère de l'Intérieur par le biais de la délivrance des passeports aux ressortissants turcs partant à l'étranger, quelle qu'en soit la cause au départ, (plusieurs types de passeports existent et leur possession n'est pas neutre), le ministère des Finances concerné par les remises des migrants (isçi dövizleri), avec, par exemple, l'existence d'une Direction des Devises Ouvrières à la Banque Centrale de Turquie (TC Merkez Bankasi) ou encore, bien qu'indirectement par ses implications induites, le ministère des Transports et des Communications, le ministère des Affaires Étrangères, celui de l'Éducation Nationale, la Direction des Affaires Religieuses du Premier Ministre... La liste est loin d'être close avec cette énumération : la Turquie est bien un pays d'émigration, mais elle n'est pas que cela.

Circulation migratoire

14 L'ensemble des circulations liées à l'émigration proprement dite ou à de nombreux flux dérivés - la circulation migratoire dans l'acception donnée par les membres de MIGRINTER (Migrations Études 1998) - peut être appréhendé par le biais de nombreuses sources documentaires et statistiques

Ce sont a priori DIE et le ministère de la Culture et du Tourisme ${ }^{6}$ qui éditent les données les plus intéressantes, mais si l'on veut approcher plus précisément la définition de la circulation migratoire, interviennent alors des organisations professionnelles et des sociétés de transport publiques ou privées (UND, UTIKAD, UATOD, UYTAD, THY, TDI...) dont les données sont centralisées au ministère des Transports. Celui-ci n'est pas toujours d'accès facile, aussi sommes-nous souvent passés directement par ces organisations professionnelles qui disposent depuis peu de sites Internet souvent très performants pour qui sait lire le turc (MIGRINTER 1997, IHESI 1999).

Immigrations en Turquie

L'immigration en Turquie est un phénomène historique ancien qui commence à se rapprocher du cas des pays d'Europe occidentale, mais qui au départ s'apparente aux flux de réfugiés et rapatriés que connurent la France coloniale (Algérie et Maghreb), l'Allemagne après 1945 (Vertriebene, Aussiedler, Übersiedler) ou la Russie aujourd'hui (de Tapia 2001, Toumarkine 1995)7. Les crises récentes de Bosnie, du Kosovo, ou l'exode en 1989 de plus de 300000 Bulgares d'origines turco-musulmanes illustrent parfaitement les mécanismes de transferts massifs de populations de terres autrefois ottomanes vers la Turquie. Cependant, actuellement, la Turquie devient terre d'immigration pour des 
expatriés fortement qualifiés autant que pour des originaires du Tiers-Monde a priori plus intéressés par l'Europe occidentale au départ (Africains de l'est, Pakistanais, voire Maghrébins!). Il convient plus dans ce cas de les définir comme migrants de transit (IOM 1995, Içduygu 1995 et 2001).

Migrations de transit en Turquie

La transition est faite avec un autre phénomène migratoire, plus proche de la mobilité que de la migration -au sens où cette migration entraînerait résidence dans un nouveau pays ou au moins une nouvelle région- phénomène fluide, contradictoire, pour lequel les appareils statistiques apparaissent peu performants: les migrations dont l'objet n'est pas la résidence dans le pays de destination. Le cas le plus simple est celui du tourisme international tel que régi par les définitions de l'OCDE, avec le passage d'une nuitée en dehors de son domicile et dans le cas présent le passage d'une frontière internationale. Ce type de mobilité intéresse aujourd'hui en Turquie de sept à dix millions de personnes par an. Les choses se compliquent singulièrement lorsque les touristes cherchent à faire du commerce non déclaré et à petite échelle (commerce de valise : bavul ticareti), lorsque les touristes se livrent occasionnellement à la prostitution (en turc, natasalar ou «les natacha", signifiant aujourd'hui prostituée d'origine slave ou européenne orientale), lorsque les touristes viennent chercher du travail en Turquie ou encore lorsqu'ils cherchent à joindre un pays tiers pour y travailler ou s'y réfugier pour des raisons politiques. La Turquie est ainsi devenue exutoire ou ballon d'oxygène pour nombre d'Irakiens, d'Iraniens (plusieurs centaines de milliers de passages des frontières certaines années), voire d'Arabes du Golfe, clairement pays de transit pour les ressortissants des pays les plus divers. L'exemple de l'Algérie est pédagogique : un Algérien peut se rendre en Turquie, principalement à Istanbul, pour les raisons suivantes :

18 - y faire du commerce et s'y installer (comme les quelques Algériens du Bazar d'Istanbul), où en l'occurrence, Istanbul semble avoir, comme Gênes ou Naples, bénéficié du retrait de la place de Marseille ;

19 - y faire du tourisme mâtiné de commerce, en fréquentant les commerçants algériens d'Istanbul (commerce à la valise, bavul ticareti) ;

20 - passer par la Turquie pour essayer de se rendre en Europe occidentale pour contourner le dispositif Schengen: la police turque arrête parfois des clandestins maghrébins sur la frontière bulgare ${ }^{8}$.

Retours et retombées économiques

21 Paradoxalement, les retours définitifs ou les retombées économiques et sociales de l'émigration dans le pays d'origine restent les plus difficiles à cerner. Nous examinerons le fonctionnement des Recensements Généraux de Population et les documents émanant d'organismes bancaires spécialisés (DESIYAB devenue Türkiye Kalkïnma Bankasï- TKB [Banque de Développement de Turquie], T.C. Halk Bankasi [Banque Populaire], TC Emlak Bankasi [Banque Immobilière], TC Ziraat Bankasï [Crédit Agricole]...) qui ont pu ou peuvent gérer des crédits pour la réinsertion des travailleurs migrants, souvent d'ailleurs en collaboration avec des dispositifs mis au point par des institutions européennes: Fonds Européen de Reconstruction, Kreditanstalt für Wiederaufbau... La principale source de données sur les retours est en Turquie la Banque Centrale qui dispose d'un service spécialisé dit "Direction générale des Devises Ouvrières ", à la base de certaines études de l'Organisation Étatique du Plan (Devlet Planlama Teskilâti). Il s'agit toutefois d'estimations sur la base des transferts de devises, 
car de fait, mis à part les services allemands de la police et de l'état-civil qui tiennent des fichiers des étrangers (inscription de la nouvelle adresse lors d'un déménagement), tous les retours non aidés par les pouvoirs publics - en France par exemple, seuls les retours aidés donnent lieu à l'ouverture d'un dossier par l'OMI - aucune source ne permet de préciser le volume des départs vers la Turquie. Cependant comme le souligne le rapport Legoux pour la DPM (Migrations Études n 95 d'août-octobre 2000), même les statistiques allemandes sont loin de répondre aux questions posées par la complexe problématique du retour.

Sources statistiques et flux migratoires : institutions et organismes de recherche publics Le ministère du Travail et de la Sécurité Sociale

Ce ministère est très naturellement le premier concerné par les flux migratoires de travail, soit parce qu'il recrute les candidats au départ, soit parce qu'il doit procéder à de complexes opérations de régularisations, ne serait-ce que pour les dossiers de sécurité sociale au retour et pour la liquidation des retraites en complément éventuel des droits acquis en Europe. Un établissement public et une direction ministérielle sont particulièrement impliqués jusqu'à la réforme de 2000 : IIBK et YIHGM.

Le bureau international du ministère du Travail est créé en 1967 (Loi 864 du 8 mai 1967 portant modification de la Loi 4841 sur la création du ministère). En 1972, ce bureau devient «Direction générale des Problèmes des Travailleurs Expatriés " (Yurtdïsï Isçi Sorunlarï Genel Müdürlügü), puis en 1983 "Direction générale des Services aux Travailleurs Expatriés » (Yurtdisï Isçi Hizmetleri Genel Müdürlügü - YIHGM) dans le cadre de la fusion des ministère du Travail et de la Sécurité Sociale. Une restructuration récente (4 octobre 2000) attribue de nouvelles missions à l'organisme qui devient "Direction générale des Relations Extérieures et des Services aux Travailleurs Expatriés » (Dïs Iliskiler ve Yurtdïsï Isçi Hizmetleri Genel Müdürlügü - DIYIH). Parallèlement, Is ve Isçi Bulma Kurumu (Office du Travail et du Placement) prend la dénomination d'Iskur (Office du Travail) et pour la première fois, l'administration décide la création d'une assurance chômage.

I.I.B.K. : Is ve Isçi Bulma Kurumu / Office du Travail et du Placement (1946-2000)

C'est un établissement public créé par la Loi 4837 de 1946 pour gérer les offres et demandes d'emploi; il cumule de fait les compétences qu'ont en France l'Agence Nationale pour l'Emploi (ANPE) et l'Office des Migrations Internationales (OMI), autrefois Office National d'Immigration (ONI) ; pour notre objet, il édite plusieurs publications spécialisées: un rapport d'activité, une revue mensuelle (Aylik Istatistik Bülteni), un annuaire statistique (IIBK Istatistik Yilligï) et des études ponctuelles.

Les annuaires IIBK sont composés d'une cinquantaine de tableaux statistiques régulièrement réédités, répartis en dix rubriques : vues générales, demandes d'emploi, offres d'emploi, placements, demandeurs d'emploi inscrits en fin d'année, travailleurs handicapés et réinsertions, services à l'étranger, statistiques générales sur les activités de l'office (en 1995: stages, cours de mise à niveau, reclassements pour cause de privatisation du secteur public ou mixte...). C'est donc ici la rubrique 8 (services à l'étranger) qui nous intéresse le plus directement.

Celle-ci comprend les tableaux suivants :

27 - demandes d'emploi à l'étranger et départs par pays et par départements turcs dans l'année, par sexe ;

- départs par pays (14) et catégories d'emploi (24 classes), par sexe ; 

suivre les tendances de l'émigration (classement des effectifs par pays de départ - c'est par exemple la source employée par Seccombe et Lawless en 1985 ou par Unbehaun, 1995 -, et de voir apparaître de nouveaux pays d'immigration (Pakistan, Israël, Roumanie, Malaisie), mais surtout de dégager des données géographiques intéressantes (classement par départements). Seccombe et Lawless ont ainsi pu déterminer que les ressortissants des départements orientaux partaient vers le Moyen-Orient arabe plus que les Turcs occidentaux plus attirés par les pays de la CEE alors que les départs actuels vers la Russie intéressent surtout des originaires des départements de la Mer Noire et d'Istanbul (ce qui de fait recoupe des habitudes anciennes, datant d'avant 1914) $)^{9}$

34 Le second tableau distingue 24 catégories de qualifications liées principalement au bâtiment-travaux publics (architectes et ingénieurs en bâtiment, dessinateurs et techniciens, chefs de chantier et contremaîtres, installateurs sanitaires et réseaux, maçons, coffreurs, charpentiers, peintres...), électriciens, monteurs et régleurs, chauffeurs et mécaniciens de chantiers, mécaniciens automobiles... et manœuvres sans qualification, généralement de l'ordre du tiers des effectifs annuels. On y apprend que les Etats-Unis recrutent surtout des cuisiniers et des garçons de café ! date du début officiel de l'émigration (prise en charge du phénomène par les pouvoirs publics) et l'année de référence de l'annuaire. Les 14 pays présents dans cette statistique incluent le Yémen, la Jordanie et le Koweït, malgré les faibles effectifs totaux et l'irrégularité des départs : ainsi le Yémen a fait appel entre 1984 et 1990 à quelques 1599 travailleurs migrants officiels pour la construction de barrages et de travaux hydrauliques. par pays et mode de sortie (par ses propres moyens, par l'intermédiaire de l'office) et origine de la société de recrutement, turque ou étrangère ; en 1995, 59483 travailleurs sont sortis de Turquie, dont 42792 pour des firmes turques et 16691 pour des firmes étrangères. On notera l'absence de complaisance de cet annuaire puisque l'on y apprend que cette année là aucune sortie n'a été organisée par le biais de l'Office! 
YIHGM : Yurtdïsï Isçi Hizmetleri Genel Müdürlügü / Direction Générale des Services aux Travailleurs Émigrés (1983-2000), actuellement DIYIH (2001-sq)

Yurtdïsï Isçi Hizmetleri Genel Müdürlügü (Direction Générale des Services aux Travailleurs Émigrés), récemment renommée Dïs Iliskiler ve Yurtdïsï Isçi Hizmetleri Genel Müdürlügü (Direction Générale des Relations Extérieures et des Services aux Travailleurs Émigrés) est un service du ministère travaillant dans le cadre de l'article 62 de l'actuelle Constitution qui prévoit que «l'État prend les mesures nécessaires pour venir en aide aux ressortissants turcs établis à l'étranger afin de garantir l'unité des familles, l'éducation des enfants, les besoins culturels et la sécurité sociale, les liens avec le pays d'origine et l'aide au retour» (O. Can Ünver YIHGM 2000: Présentation du rapport 1999 par le Directeur Général).

Des délégations sont installées dans une quinzaine de pays d'immigration aux échelons des Ambassades et Consulats Généraux et les Attachés du Travail des Ambassades adressent depuis 1979 des rapports sur la situation de l'émigration et les problèmes rencontrés dans la défense des droits des ressortissants turcs établis à l'étranger. Ces rapports « nationaux » sont ensuite condensés en un volume diffusé à divers acteurs et institutions intéressés par le sujet: ambassades et représentations consulaires, organisations patronales et syndicales, organes de presse, universités et organismes de recherche. Ce rapport annuel est précieux pour les possibilités de recherche comparative qu'il offre.

Certes, tous les pays ne sont pas traités de la même manière, en raison de différences substantielles quant à la collecte des renseignements statistiques, voire à leur présence ou absence, mais le rapport veille à garder un plan cohérent dans l'exposé des faits, que l'on soit en France ou en Libye ! Le plan habituel est ainsi organisé :

40 - Première partie : répartition des travailleurs et ressortissants turcs en émigration, éléments généraux (récapitulatifs généraux, sorties annuelles...)

41 - Seconde partie : rapports par pays (Allemagne, Pays-Bas, Belgique, France, Danemark, Autriche, Suisse, Suède, Grande-Bretagne, Australie, Koweït, Libye), avec les rubriques suivantes :

42 - Indications démographiques,

43 - Données relatives au travail et à l'emploi,

44 - Sécurité Sociale,

45 - Education,

46 - Organisations de travailleurs (associations),

47 - Réglementation et jurisprudence nationale,

48 - Économie générale du pays d'immigration.

49 - Troisième partie : éléments de législation, réglementation, jurisprudence internationales

50 - Quatrième partie : évolutions des législations et réglementations turques

51 - Cinquième partie : organisation et activités de l'établissement

52 La première partie est très courte et consiste principalement en quelques tableaux généraux: ressortissants turcs et main-d'œuvre à l'étranger par pays, départs dans l'année par pays et par sexe, répartition par mois de départ, quelques courts tableaux de récapitulations sur les dernières années. 
53 La seconde partie est fondamentale pour l'étude géographique du champ migratoire, particulièrement pour les effectifs résidant dans chaque pays détaillés par circonscriptions administratives (département français, Land allemand et autrichien, canton suisse, province ou autre ailleurs). Pour quelques pays, la répartition descend parfois jusqu'à la ville (municipalité) dans laquelle résident plus de 10000 habitants d'origine turque (YIHGM 1991), voire 5000 (YIHGM 1994). Ceci permet une cartographie déjà relativement affinée de la présence turque en Europe, en tous cas plus précise que celle permise par Eurostat et SOPEMI. Il est nécessaire de passer par les statistiques nationales en l'absence de ce rapport. Suivent des données diversifiées, mais difficilement comparables d'un pays à l'autre sur les titres tels que l'éducation, la sécurité sociale, les salaires pratiqués... La lecture du rapport permet cependant une vision globale - mais très partielle -, à l'échelle du champ, de l'importance de la création d'entreprise, du tissu associatif (une simple estimation du nombre des associations dans chaque pays).

La troisième partie traite des relations internationales, entre ministère du Travail et organismes internationaux ou nationaux compétents en matière d'emploi et de migration de main-d'œuvre. Dans le rapport 1997 (YIHGM 1998), par exemple, il est dit que la Turquie a signé 15 accords avec le Bureau International du Travail, a participé aux conférences plénières et restreintes de l'organisation de Genève, ainsi qu'aux Comités de spécialistes du Conseil de l'Europe (Strasbourg), mais aussi aux instances de l'OCDE à Paris, au traité d'Amsterdam, au sommet de Luxembourg, à divers séminaires et conférences internationales. Les sujets traités sont classiques, ardus, généralement assez peu connus de la plupart des chercheurs sur les migrations, à l'exception des juristes et des spécialistes du champ politique. La collection YIHGM est donc une source de premier plan pour l'étude des évolutions juridiques et politiques de l'émigration turque. Ce d'autant plus que la description commentée des travaux menés avec les instances internationales est suivie des analyses de grands problèmes d'actualité (libre circulation des travailleurs, politiques migratoires et pays tiers, protection sociale et égalité des droits, politique envers les travailleurs handicapés, lutte contre le racisme, système statistique européen, santé et sécurité, normes de travail, dialogue social, égalité des sexes, fonds structuraux...) avec les principaux pays employeurs de maind'œuvre immigrée turque, de plus en plus souvent remises dans leur contexte européen, et un résumé détaillé, acte par acte, de la jurisprudence relative à des plaintes déposées en Europe par des travailleurs turcs (pour notre année de référence, tribunaux de Berlin, Bonn, Munich, Stuttgart, Darmstadt, Cologne, Nuremberg...). Ce chapitre se termine par une information sur divers accords bilatéraux relatifs à la non double imposition de ressortissants turcs résidant dans divers pays d'Europe centrale et orientale comme d'Asie centrale.

55 La quatrième partie passe en revue les évolutions des réglementations turques en matière de travail, migration de main-d'œuvre, retour et retraite, importations en douane... On y trouvera aussi bien les grilles indiciaires permettant les calculs des droits à la retraite que les listes de matériels admis à l'importation par les migrants résidant à l'étranger, en cas de congés ou de retour définitif. Il s'agit là d'un point sur l'évolution de l'année écoulée ; YIHGM et TCMB (la Banque Centrale de Turquie) éditent des guides très complets sur l'état des réglementations en vigueur.

La cinquième partie relève du rapport interne d'activité et elle est suivie d'une annexe donnant pour 1997 les coordonnées des bureaux de liaison à l'étranger. Ce rapport 
interne est celui d'une administration tournée entièrement vers l'étranger: activité mesurée à la production d'archives ou d'actes juridiques, état des personnels détachés à l'étranger, budget. La cartographie que l'on pourrait établir avec l'annexe laisserait paraître ce que les Turcs nommait autrefois le $68^{\text {ème }}$ département (68. Vilâyet), la Turquie émigrée, avec son armature administrative.

L'intérêt majeur de cette collection - on peut parler de littérature grise, mais de fait facilement accessible puisque l'on peut se procurer les rapports sur simple demande écrite - est de fournir une vision d'ensemble de 1979 à aujourd'hui et de mesurer les évolutions sur cette période cruciale où le champ migratoire change profondément ; on y voit apparaître les pays de la CEI et en particulier ceux d'Asie Centrale, mais on y voit aussi les évolutions des relations Turquie - CEE jusque dans les jurisprudences nationales en matière de droits des travailleurs émigrés. La complexe réglementation turque y figure aussi et comprend aussi bien les changements intervenus sur les régimes de retraite que ceux en vigueur sur l'importation de véhicules automobiles par les migrants turcs. Depuis peu, ce rapport est en partie édité sur Internet, consultable sur le site du ministère du travail et de la sécurité Sociale : http://www.calisma.gov.tr Le Premier Ministre et les délégations générales du Premier Ministre Plusieurs délégations sont rattachées à la Présidence du Conseil et doivent lui apporter un concours technique sur des dossiers d'aide à la décision. Pour ce qui concerne notre objet, trois Directions Générales sont compétentes indirectement en matière de mobilité et migrations internationales : la Direction générale de l'Institut National des Statistiques (DIE), la Direction générale de l'Organisation d'Etat à la Planification (Devlet Planlama Teskilâtì), la Direction générale des Affaires Religieuses (Diyanet Isleri Genel Müdürlügü). Deux de ces organismes sont régulièrement consultés, le troisième ne l'est pas, à notre connaissance, mais il serait utile de prendre contact car il possède certainement ses propres statistiques. Du premier ministre dépend également une Antenne d'Information sur l'Entrepreneuriat du secteur du Bâtiment et des Travaux Publics à l'Étranger (YMHBBD - Yurtdïsï Müteahhitlik Hizmetleri Basbakan Bas Danismanligi).

DIE - Devlet Istatistik Enstitüsü / Institut National des Statistiques

59 Cet établissement joue un rôle analogue à celui de l'INSEE pour la France. Établi à Ankara, il fonctionne depuis les années 1930 - le premier recensement au sens moderne date de 1927 - et publie de très nombreux fascicules, annuaires ou études statistiques consultables au centre de documentation DIE sur place, en vente sur place ou par correspondance ou mis à disposition gratuite pour les institutions répertoriées. DIE édite des documents bilingues turc-anglais et propose aujourd'hui un site Internet (http://www.die.gov.tr). S'il n'existe pas de statistiques propres à l'émigration, celle-ci est présentée sous de multiples formes.

Dans les annuaires statistiques

60 - le chapitre « statistiques vitales" présente les immigrants par pays d'origine, les réfugiés d'origine turco-musulmanes (dont la vocation reconnue est l'intégration en Turquie sur le modèle des Aussiedler en Allemagne), les demandeurs d'asile par pays d'origine (les réfugiés au sens plus classique du terme, certains pouvant à terme s'installer en Turquie), les étrangers résidant en Turquie par nationalité et objet du séjour - résidence, éducation, travail, autres - et de ce fait possesseur d'une carte de séjour (ikamet tezkeresi). Ces tableaux édités par DIE sont fournis par la Direction Générale des Services Ruraux (Köy Hizmetleri) et la Direction Générale de la Sécurité 
(Emniyet). En d'autres termes, c'est la Turquie, pays d'immigration qui apparaît ici avec des flux de nature très différentes :

61 - l'immigration des rapatriés et réfugiés, composante de l'ethnogenèse turque, assimilant des Turcophones ou des Turcs ethniques (des Tatars aux Kazakhs du Xinjiang et aux Kirghizes du Pamir afghan) et de nombreuses populations entrées en contact avec l'empire ottoman: Balkaniques (Serbes islamisés, Bosniaques, Albanais, Bulgares islamisés, Tziganes...), Caucasiens d'origines très diverses... Cette immigration très étudiée en Turquie (Dumont, Kazgan, Karpat, Simsir, Svanberg, Dor, Toumarkine...) a touché une population de l'ordre de sept millions de personnes entre 1771 et aujourd'hui et entre dans la composition ethnique de l'actuelle Turquie ; ils gardent si possible un lien avec les membres de l'ethnie restés en arrière, dans le pays d'origine (Andrews et alii 1989).

62 - La Turquie, pays d'immigration, est aussi un pays qui commence sérieusement a attirer les cadres et les agents qualifiés des multinationales, par le biais de l'investissement privé. Par contrecoup et par contraste avec les pays voisins ou plus lointains (Asie, Afrique orientale), la Turquie devient attractive pour son niveau de vie relativement élevé et sa position de contact entre Orient et Occident. Les statistiques DIE nous renseignent avant tout sur la migration légale des étrangers venus travailler en Turquie.

63 - La migration de transit peut apparaître dans le détail des nationalités concernées par le tableau intitulé «Étrangers se trouvant en Turquie par nationalités et motifs de séjour ». Ainsi en 1996, 106473 étrangers détiennent des cartes de séjour. Sur ce nombre, on relève 7284 Américains, 5415 Allemands, 2400 Français et 994 Japonais, mais aussi 2695 Afghans, 4369 Iraniens et 1925 Irakiens. 48 nationalités sont présentes (dont l'URSS et la CEI), mais aussi des apatrides dits Heimatlos ou Gayri Muntazam (la différence entre les deux catégories n'est pas claire, car elles portent en sous-titre, vatansiz: apatride), les réfugiés sont de fait comptés ailleurs (Immigrants par pays d'origine et réfugiés par pays d'origine $)^{10}$. Le faible nombre d'Iraniens ou d'Irakiens ne doit pas faire illusion, il s'agit bien de résidants ayant une carte de séjour et non d'entrées en Turquie : ce chiffre est à relever sur les statistiques du tourisme.

64 - Le chapitre "sécurité sociale" présente les activités de l'IIBK en Turquie et à l'étranger. Pour l'étranger, il s'agit des placements par sexe, groupe de qualification (qualifiés, non qualifiés), qualifications. Elle présente également les activités du Croissant Rouge (Kïilay : crédits affectés aux réfugiés-rapatriés, göçmen),

65 Pour l'année 1995 (avec reprise de la période 1989 - 1995) éditée en 1996, sont distinguées les destinations suivantes : Allemagne, Aruba (Antilles), Australie, Autriche, Émirats, Afrique du Sud, Irak, Suisse, Chypre-Nord, Koweït, Libye, Jordanie, ex-URSS, Arabie Saoudite, Yémen, autres pays (dans l'ordre alphabétique en turc). Les 24 catégories professionnelles sont les mêmes que dans l'annuaire IIBK. Cette source, peut-être plus accessible qu'IIBK, n'a pas d'autre intérêt pour notre étude que celle de cette accessibilité pour qui n'est pas connaisseur de l'organigramme des administrations turques et surtout du contexte très varié et très riche relaté par ce document de 700-800 pages sur l'économie du pays.

66 - Le chapitre " tourisme " est intéressant car il permet de suivre l'évolution globale de la stratégie d'ouverture de la Turquie. Nous disposons de plusieurs tableaux généraux permettant de suivre l'évolution des entrées et sorties des étrangers et des nationaux par années, mois, motifs de déplacement, modes de transport, départements 
de sortie du territoire (regroupement des douanes par départements), pays de destination, à laquelle s'ajoute un tableau sur le bilan de la balance touristique. Ces données sont déjà relativement riches pour ce qui est des passages aux frontières, mais les fascicules "tourisme" sont, nous le verrons, encore plus précis pour certaines années où des traitements plus fins sont édités $(1990,1995,1995)$.

- Le chapitre "argent, banque" présente un tableau des entrées mensuelles des devises ouvrières (isçi dövizleri $=$ remises des migrants).

Enfin, le chapitre «balance des paiements » permet de saisir la place des remises des migrants, des transferts des réfugiés en transferts privés (özel) et publics (resmî).

Dans les fascicules spécialisés

69 - Les fascicules départementaux des recensements généraux de la population comportent une rubrique "résidant à l'étranger au moment du recensement " ainsi qu'une rubrique " résidant à l'étranger lors du dernier recensement »; ceci permet une estimation de la présence départementale à l'étranger (émigration) et une estimation du volume des retours sur la période intercensitaire. Mais ces deux données restent partielles puisque dépendant des répondants au recensement : les familles absentes à ce moment ne répondront évidemment pas.

70 - Les fascicules tourisme, comportent les entrées et sorties de ressortissants turcs par motifs de sortie, voire parfois comme dans les éditions 1990 et 1995, un tableau permettant de dissocier résidents en Turquie et résidents à l'étranger, autorisant l'estimation relativement cohérente des flux liés à la circulation migratoire. L'analyse à moyen terme des destinations déclarées par les nationaux turcs sortant du territoire permettra de saisir l'évolution des trafics dans les Balkans et vers l'Europe orientale. Pour 1995, nous disposons des tableaux suivants :

71 - nationaux turcs par département de sortie (poste frontalier), mode de transport, mois (1995 : résidents en Turquie et à l'étranger)

72 - nationaux turcs par département de sortie (poste frontalier), mode de transport, mois (1995 : résidents en Turquie)

73 - nationaux par catégories professionnelles et motifs de sortie (1995: résidents en Turquie)

74 - nationaux par pays de destination et motifs de sortie (1995 : résidents en Turquie)

75 - nationaux par département de résidence, groupe d'âge et sexe (1995: résidents en Turquie)

76 - nationaux par pays de destination, catégories professionnelles et sexe (1995: résidents en Turquie)

$77 \mathrm{Au}$ total, trente-trois tableaux sont ainsi mis à disposition. Pour l'objet de l'étude, ce sont évidemment les tableaux indiquant les pays de destination et les tableaux relatifs aux résidents à l'étranger qui nous intéressent. Les tableaux donnant le détail des pays de destination des ressortissants turcs distinguent onze rubriques : pays européens de l'OCDE, autres pays de l'OCDE, autres pays européens, Etats de la CEI, Asie orientale, Asie du Sud-Est, Asie du Sud, Asie occidentale, Afrique, Amérique (hors OCDE), Océanie (hors OCDE), soit au total 99 pays.

78 On apprend ainsi que 53201 ressortissants turcs ont fait en 1995 le pèlerinage de la Mecque, que 25839 se sont rendus en Arabie saoudite pour y travailler, que sur 15600 militaires en mission à l'étranger, 11997 se sont rendus à Chypre, 929 aux USA, 46 en 
Israël, 1051 en Italie, 379 en Allemagne. Pour l'Azerbaïdjan, sur 52746 départs, 5653 répondaient à des motivations touristiques, 2030 à des raisons familiales, 1584 étaient des déplacements liés au travail et 24618 aux affaires !

Pour les «pays de transit» vers l'Europe occidentale, i.e. le groupe "Autres pays d'Europe ", ce ne sont pas moins de 361199 personnes qui ont fait un ou plusieurs voyages à destination de l'un de ces pays, principalement en Bulgarie (245 992) et Roumanie (70 572). Ces chiffres sont à comparer d'une part avec les départs vers la Russie (120 218) et d'autre part avec les statistiques donnés par la police bulgare pour le transit turc. Il convient d'y rapporter le chiffre des Turcs sortant de Turquie et résidant en Bulgarie selon leurs déclarations à la douane turque (Yurtdisïnda ikamet eden: résidant à l'étranger, pays : Bulgarie), soient 20001 sorties pour 1995. Un peu moins de $10 \%$ des sorties vers la Bulgarie seraient donc le fait de résidents. Dans un cas comme dans l'autre, il faudra se rappeler que la même personne peut se déplacer plusieurs fois dans l'année. Le rapport OIM « Transit Migration in Bulgaria » (1994) nous signale ainsi 3950000 transits de ressortissants turcs en 1990 pour seulement 1580000 en 1993. La route des Balkans a de fait subi de plein fouet la crise yougoslave et si deux routes alternatives sont très vite apparues, dont l'une par Budapest, Bucarest et le pont de Giurgiu sur le Danube passe effectivement par le territoire bulgare, ce sont surtout, sur la route adriatique, les car-ferries grecs et turcs, et encore plus l'avion, qui bénéficient de ces réajustements (MIGRINTER 1997 et 1999).

Il ne s'agit là que d'un très modeste aperçu de ce que l'on peut tirer des statistiques turques du tourisme, rassemblées de fait par la police des frontières depuis 1992-93. Cette série d'indicateurs est intéressante, mais demande à être sérieusement examinée de façon critique, car il y a bien là passage de frontière internationale, mais de la frontière turque uniquement. Il serait utile de comparer si possible avec les statistiques équivalentes des pays d'Europe orientale.

81 - Les fascicules "commerce extérieur", dont nous disposons, publient des statistiques très complètes sur les échanges internationaux de l'économie turque avec le reste du monde. Les treize tableaux de la série (DIE 1997, paru en 1998, $\mathrm{n}^{\circ} 2212$ ) couvrent les données suivantes:

- Importations, exportations et indicateurs choisis, 1923-1997

- Commerce extérieur par mois, 1992-1997

84 
Emmanuel Ma Mung et nous-même à partir des chiffres rassemblés par le ministère français des Transports (SITRAM) nous a montré que cette batterie d'indicateurs était assez difficile à analyser du fait des catégories statistiques internationales (ISIC et SITC). Il s'agit bien là de démarquer ce qui est le fait de la circulation migratoire et de la desserte de populations émigrées et ce qui ressort du commerce international entre états et économies nationales. Nous pensons toutefois qu'il y a là pour les économistes et les géographes des flux une piste de recherche à envisager sérieusement. Des analyses fines sur la consommation des groupes sociaux (ex les immigrés de Turquie face aux travailleurs nationaux, à revenus égaux) à partir de certaines catégories de produits importés pourraient être intéressantes. Une catégorie spécifique de produits importés peut révéler la présence d'une catégorie spécifique de clientèle (commerce ethnique, ethnic business, halal business $\left.{ }^{11}\right)$. globales. Elle va cependant se révéler fort utile pour l'étude des routes du commerce international turc et les destinations géographiques des transporteurs de marchandises. Elle sera alors à comparer avec les statistiques de transports de personnes dont nous disposons avec le tourisme international. Les données ici presque toutes quantitatives peuvent être systématiquement confrontées aux données à la fois quantitatives et qualitatives que publient les transporteurs eux-mêmes ou le ministère turc des Transports.

Devlet Planlama Teskilâti, l'Organisation Étatique de Planification, State Planning Organization produit pas à proprement parler de statistiques, mais elle les étudie, parfois avec beaucoup de soins. C'est cette organisation dépendant du Premier Ministre qui a ainsi édité de nombreuses études d'excellente qualité sur la migration internationale de travail (Nermin Abadan dès 1965, la première d'une longue série de références turques sur le sujet), sur la migration interne, l'évolution démographique, ainsi que de très nombreux rapports de conjoncture sur la population, la production industrielle, l'aménagement du territoire, les transports, les communications... C'est aussi à DPT que travaillait Mme Ayfur Barisik, la rédactrice du rapport SOPeMi, aujourd'hui remplacée par le Professeur Ahmet Gökdere, spécialiste de l'émigration et des ses retombées économiques et sociales.

104 L'une des sources possibles dans l'étude de la circulation migratoire est par exemple l'existence de rapports de commissions ad hoc sur l'un ou l'autre aspects de l'économie 
turque. Nous pensons ici à la diffusion en 1989 et 1995 de deux séries de rapports détaillés sur l'évolution des modes de transports en Turquie, avec dans chaque cas la prise en compte des relations internationales (transports TIR, autocars, compagnies aériennes). Les rapports remis au Parlement à l'occasion de discussions précises - loi sur la planification, les plans de développement, lois de finances, discussion des budgets..., donnent lieu à l'édition de rapports spécifiques que l'on peut consulter dans les bibliothèques ouvertes au public (DIE, DPT, TOBB - Union des Chambres et Bourses de Commerce et d'Industrie, CCI départementales - celles d'Istanbul, Izmir ou d'Ankara ont des fonds documentaires dignes d'intérêt...) ou se procurer directement en fonction des tirages, malgré tout, souvent limités.

Köy Hizmetleri Genel Müdürlügü / Direction Générale des Affaires Rurales

Citée pour mémoire, cette Direction gère en particulier l'intégration des réfugiésrapatriés turco-musulmans admis collectivement en Turquie selon un modèle, nous l'avons déjà souligné, proche de celui des Aussiedler allemands. Cette fonction d'établissement (iskân göçmen $=$ muhacir ) va jusqu'à la construction de villages de réfugiés en Anatolie, comme cela a été réalisé pour les Kazakhs du Xinjiang entre 1954 et 1960 ou les Kirghiz du Pamir afghan après 1981, cités ci-dessus. La bibliographie turque (histoire, sociologie, ethnologie, géographie...) comprend là aussi des milliers de références sur les populations accueillies dans l'empire, puis la république turque: Albanais, Bosniaques, Turcs des Balkans, de Chypre, de Crête, de Hongrie, Tatars de Crimée (et même un petit groupe de Sibériens d'origine ouzbèke !), Caucasiens...

Diyanet Isleri Baskanligi / Présidence des Affaires Religieuses

Cette Présidence (que l'on pourrait traduire en français administratif par Délégation Générale) gère les personnels religieux musulmans sunnites qui peuvent être amenés à desservir les lieux de culte établis à l'étranger. Nous la citons pour mémoire car très peu connue, à notre connaissance non étudiée par son rôle à l'étranger, elle doit cependant posséder ses propres statistiques, en raison de la présence dans les Consulats d'attachés sociaux qui en sont issus et qui supervisent les activités des imams desservant les communautés groupées en associations dans toute l'Europe dans une fédération nommée DITIB en Allemagne. Elle mériterait donc une étude particulière (voir le site: http://www.diyanet.gov.tr pour la présentation générale de la Présidence).

YMHBBD : Yurtdïsï Müteahhitlik Hizmetleri Basbakan Bas Danïsmanlïgï ou YMHBBM Yurtdïsï Müteahhitlik Hizmetleri Basbakan Bas Müsavirligi / Délégation des Services du secteur Bâtiment-Travaux publics auprès du Premier Ministre

Ce bureau d'information, lui aussi rattaché au Premier Ministre, recense les données sur les activités des entrepreneurs du BTP à l'étranger. Rassemblant une centaine de grandes entreprises, le secteur qui dispose par ailleurs d'une Union patronale spécifique (TMB-Türk Müteahhitler Birligi : Union des Entrepreneurs Turcs du Bâtiment et des Travaux Publics, à Ankara) a rapporté au moins à la balance des paiements 33 milliards US \$ entre 1974 et aujourd'hui (chantiers en cours pour une quinzaine de milliards \$). 20 à 40000 travailleurs sont chaque année concernés par ce secteur d'activité, mais il n'est pas rare que des entreprises étrangères viennent renforcer ce courant en recrutant directement des travailleurs en Turquie, comme dans le cas déjà cité du chantier d'Aruba.

Le Premier Ministre dispose enfin d'un établissement public (TIKA) créé pour organiser les activités de coopération internationale dans le monde turco-musulman lato sensu, 
établissement qui dans certains cas peut diffuser des informations intéressantes sur l'Asie Centrale ou les Balkans. Ce rattachement est récent, aussi TIKA sera évoqué infra, dans le cadre initial du ministère des Relations Extérieures.

Le ministère de l'Intérieur

Ce ministère occupe une place particulière dans la gestion des flux migratoires. L'une de ses missions est évidemment de délivrer, par l'intermédiaire des préfectures et souspréfectures, les papiers d'identité, passeports en général, qui permettent de partir à l'étranger.

Emniyet Genel Müdürlügü / Direction générale de la Sécurité

110 La Direction Générale de la Sécurité, compétente en matière de police, intervient pour ce qui nous concerne, dans divers secteurs liés aux migrations internationales: délivrance de passeports aux ressortissants turcs et aux réfugiés ayant vocation à s'établir en Turquie (iskân göçmen pris en charge par la Direction des Services Ruraux Köy Hizmetleri), de cartes de séjour (ikamet tezkeresi) aux ressortissants étrangers. C'est elle aussi qui depuis 1992-93 fournit les statistiques de passages aux frontières traitées par DIE et le ministère du Tourisme.

Les délivrances de passeports aux ressortissants turcs

$111 \mathrm{Au}$ moment du déclenchement de l'émigration, la possession d'un passeport était peu courante. Au-delà des passeports diplomatiques, la circulation à l'étranger des nationaux turcs n'était guère facile ; il aura fallu attendre les années quatre-vingts pour qu'apparaissent avec l'élévation du niveau de vie de certaines catégories de populations un véritable tourisme international turc. Les difficultés économiques et le contrôle des changes allaient également dans le sens des restrictions de sortie. Aussi la possession d'un passeport touristique (pour se rendre à l'étranger et chercher du travail) ou d'un passeport de travailleur émigré (donnant droit à une réelle liberté d'entrer et sortir du territoire) était un avantage certain et précieux. La couleur du passeport comme les mentions apparentes (passeport de service, travailleur [isçi], membre de famille de travailleur [isçi ailesi üyesi]) ne sont toujours pas anodines car, si la Turquie a largement libéralisé ses pratiques, l'Europe n'est toujours pas prête à faire bénéficier les migrants ou les immigrés d'origine turque de la libre circulation.

Les entrées de réfugiés en Turquie

112 Il convient ici de distinguer réfugiés-rapatriés (muhacir / göçmen) ayant vocation à s'installer en Turquie parce que d'origine turco-musulmane et réputés facilement assimilables et réfugiés " humanitaires ", selon la Convention de Genève, pour lesquels les traitements sont assez mal définis (mülteci, iltica). Appartiennent à cette dernière catégorie Iraniens en transit, Irakiens d'origines chiite, kurde, assyro-chaldéenne, Afghans, opposants d'origines diverses tolérés et en attente de clarification des relations bilatérales (Ouzbeks, Tunisiens, ressortissants de pays balkaniques...). Les flux d'entrée sont parfois considérables : plus de 80000 Kurdes irakiens après Halabja, plus de 600000 après la guerre du Golfe, plus d'un million d'Iraniens ayant transité par la Turquie, plusieurs dizaines de milliers de Bosniaques et de Kosovars, mais il est difficile de faire la part entre les diverses catégories de réfugiés. Ainsi Bosniaques ou Kosovars sont admis comme réfugiés temporaires et rejoignent souvent leurs familles déjà établies, se fondant dans la catégorie des réfugiés-rapatriés à titre individuel (serbest göçmen $)^{12}$. Kazakhs du Xinjiang (1954-1962) ou Kirghizes du Pamir afghan (1981) ont été admis comme réfugiés collectifs (iskân göçmen) pour lesquels des villages ont été 
construits tandis que les Kazakhs d'Iran ou d'Afghanistan parvenus en Turquie individuellement ou en famille étaient considérés comme serbest göçmen.

Les délivrances de titres de séjour aux étrangers résidant en Turquie

Elle s'apparente à la gestion des cartes de séjour européennes et les données sont publiées dans les annuaires DIE. Depuis les années 1990, avec la croissance des capitaux étrangers investis en Turquie, cette activité a notablement augmenté.

Hormis ces statistiques du domaine public, éditée par DIE chaque année, la Direction Générale de la Sécurité tient à jour d'autres séries plus confidentielles. La presse peut cependant en publier l'une ou l'autre, comme les résultats de la surveillance des frontières avec le nombre de passages clandestins ayant donné lieu à arrestation des contrevenants. Contact a été pris dans le cadre du rapport IHESI (1999) avec l'Attaché de Police de l'Ambassade de France ainsi qu'avec le Directeur des Relations Internationales de la Direction Générale à Ankara, puis avec le responsable de la Direction des Étrangers et des Frontières ${ }^{13}$. Il en ressort que deux Directions sont susceptibles de fournir des statistiques intéressant notre objet :

115 - le Bureau des Etrangers et des Frontières (Yabancilar ve Hudut Daire Baskanligi),

116 • le Bureau de la Lutte contre l'Immigration Clandestine, la Contrebande et le Crime Organisé (Yasadisï Göç, Kaçakçilik ve Organize Suçlarla Mücadele Daire Baskanlïgi).

117 A la suite de conversations téléphoniques et d'échanges de fax, la Direction des Relations Internationales nous a fait parvenir deux tableaux de synthèse portant sur la période 1995-1000, l'un sur les arrestations de migrants clandestins, l'autre sur les réfugiés dont le transit était autorisé.

118 - En 1995, 3591 migrants en situation irrégulière ont été arrêtés par la police turque, 26331 au 23.11.1999, soit au total 65690 sur la période considérée, ce chiffre augmentant très considérablement en 1999-2000. Ainsi durant l'année 2000, le nombre de personnes appréhendées par la police atteint 31399 en territoire turc, 94514 sur les frontières internationales, soit un total de 125913.

119 • le nombre de réfugiés admis à transiter par la Turquie vers un pays d'accueil tiers est pour une période équivalente (de 1995 au 01.11.1999) de 5 761. En grande majorité Iraniens (3 270) ou Irakiens (2 425), ces réfugiés se sont dirigés principalement vers les États-Unis, le Canada et les pays scandinaves.

120 Ces premiers contacts comparés à ce que l'on connaît par ailleurs et qui relève de données non confidentielles permet d'augurer de l'existence d'un appareil statistique plutôt performant pour l'objet de notre réflexion.

Le ministère des Affaires Étrangères

$121 \mathrm{Au}$ vu de certaines données fragmentaires apparaissant dans des publications publiques (DIE, YIHGM, IIBK, TIKA...) ou privées (DEIK), il apparaît que le ministère des Affaires Étrangères de Turquie possède des statistiques intéressantes.

TIKA : Türk Isbirligi ve Kalkïnma Ajansi / TICA :Turkish International and Cooperation Agency

122 Cet organisme (http://www.tika.gov.tr) est un établissement public récent, dépendant à sa création du ministère des Affaires Étrangères (en turc: Relations Extérieures, Dïs Iliskiler Bakanligi) dont les missions sont le développement et la coopération culturelle, technique, économique et scientifique envers des pays partenaires ayant des liens «naturels» avec la Turquie, à savoir au départ les républiques turciques (Türk̂̂ 
Cumhuriyetler, souvent simplement dites Türk Cumhuriyetleri ou républiques turques) et les pays à populations turco-musulmanes (Chypre-Nord, Caucase, Russie turcophone : Tatarstan, Tchouvachie, Bashkirie...).

Il est aujourd'hui rattaché au Premier Ministre, mais nous avons préféré le garder, pour la période qui nous intéresse le plus directement, à cette place. Avec le temps, la zone de compétence de TIKA s'est étendue aux pays riverains de la Mer Noire, à ceux des Balkans, à la Mongolie..., dans les domaines les plus divers. Le trait commun de l'ensemble de ces pays reste toutefois une relation privilégiée, soit linguistique, soit religieuse, soit encore culturelle et historique avec des Etats qui ont fait partie de l'empire ottoman ou appartiennent au monde turcophone (Türk Dünyasi). Les relations avec la lointaine Mongolie passent par la présence d'une population kazakhe restée musulmane et l'existence dans ce pays de monuments épigraphiques turcs $\mathrm{du} \mathrm{VI}^{\mathrm{e}}$ au $\mathrm{IX}^{\mathrm{e}}$ siècles, fondateurs de la littérature turque. Le Président Süleyman Demirel s'est rendu sur le site et la Turquie finance la conservation des sites archéologiques. On notera une certaine désaffection de TIKA face au Tadjikistan iranophone - qui peut cependant s'excuser par l'atmosphère de guerre civile que connaît ce pays depuis déjà presque dix années - et l'absence de relations avec les pays arabes.

124 TIKA publie de nombreux documents destinés aux décideurs turcs, mais aussi au public étranger en relation avec l'objet de son apparition : l'émergence possible d'un monde turcophone plus proche de la définition économique et culturelle de la francophonie que des vieux rêves pan-touranistes ou pan-turquistes que certains observateurs extérieurs seraient tenté de déceler dans l'actuelle politique turque. Ces documents ou collections sont les suivants :

125 - la revue Eurasian Studies, en turc Avrasya Etüdleri, revue de fond ouverte aux auteurs étrangers de la zone TIKA, mais aussi aux turcologues et politologues occidentaux; parmi les auteurs américains : Paul Henze, Albert Wohlstetter, Zbigniew Brzezinski, Enders Wimbush... ${ }^{14}$,

126 - le bulletin Eurasian File, en turc, Avrasya Bülteni, bulletin d'information intéressant directement notre objet dans la mesure où il donne de fréquentes informations sur les chantiers et les entreprises turcs travaillant dans les pays de la zone TIKA,

127 - les rapports par pays (Ülke Raporu), parfois accompagnés de volumes spécifiques sur les réglementations (Mevzuat) développent, parfois annuellement, les informations données par les bulletins; il s'agit là de véritables guides à l'usage des entrepreneurs ou hauts fonctionnaires turcs amenés à se rendre en Asie Centrale d'abord, dans toute la zone TIKA ensuite, pour y travailler. Pour notre objet, il conviendra donc de consulter les volumes "Bosnie-Herzégovine ", "Albanie ", " Macédoine »..., à rapprocher des travaux de la Chambre de Commerce d'Istanbul (ITO) présentés par Samim Akgönül dans le rapport IHESI (1999). Les volumes de réglementation donnent les références de tous les textes législatifs, réglementaires, bilatéraux ou multilatéraux, utiles aux entrepreneurs et investisseurs turcs (non double imposition, régimes fiscaux, douaniers, ...

128 L'émigration n'est pas l'objet premier des travaux de TIKA. On retrouve cependant vite des données la concernant par le biais des relations économiques denses créées depuis peu avec certains pays: Azerbaïdjan, Kirghizie, Turkménistan, Kazakhstan, Ouzbékistan, Albanie, Bosnie, Géorgie, Roumanie, Ukraine, ... ou Russie. C'est par le canal des investissements turcs dans ces pays, le plus souvent en financement croisé 
(joint venture), la mise en œuvre de chantiers de travaux publics, des opérations humanitaires, une politique culturelle volontaire (qui est autant l'œuvre du secteur privé que des pouvoirs publics) que les Turcs s'installent comme cadres, techniciens, conseillers techniques, enseignants, transporteurs et surtout commerçants. Il semblerait que les döner kebap déjà intégrés dans les villes d'Europe ou d'Australie aient commencé leur installation... en Mongolie! TIKA nous donne dans ses bulletins ou ses rapports par pays des listes d'entreprises à capitaux turcs présentes aussi bien dans les Balkans et en Europe centrale qu'en Asie centrale.

es données qui restent toutefois fragmentaires peuvent être recoupées avec les chiffres annoncés par YIHGM dans ses derniers rapports, mais aussi par les documents professionnels émanant du secteur marchand privé (compagnies consulaires, transporteurs).

YIHGM (1998) pour l'année écoulée (1997) fait état de 41570 ressortissants installés dans les pays de la zone TIKA (Azerbaïdjan, Biélorussie, Géorgie, républiques centreasiatiques, dont 300 au Tadjikistan, Moldavie, Russie devenue la première destination de l'émigration turque depuis les années 1990, et Ukraine. TIKA, en particulier dans ses bulletins et ses rapports, donne des informations sur les entreprises turques installées dans les pays de sa zone de compétence (typologie, raisons sociales, implantations, effectifs).

Au-delà des chiffres et des tableaux ou des listes plus qualitatives, TIKA reprend périodiquement la liste des réglementations ou des accords internationaux allant dans le sens de la coopération définie ci-dessus. En matière de migration (émigration, immigration, transit), ce sont par exemple les accords portant sur la non double imposition des ressortissants des pays d'origine / de résidence, les accords sur les transferts de capitaux ou de bénéfices, les accords bilatéraux sur les visas, les quotas autorisés pour les transports en transit ou à destination des parties signataires... Il ne s'agit certes pas de migration à proprement parler, mais à chaque fois de mesures fiscales, économiques, réglementaires..., facilitant la circulation des hommes, des capitaux et des marchandises.

Le ministère des Finances et la Banque Centrale de Turquie

La TCMB - Türkiye Cumhuriyeti Merkez Bankasi / Banque Centrale de la République de Turquie, était en théorie seule habilitée au transfert des devises ouvrières (isçi dövizleri), avec un taux de change plus favorable que le taux commercial ou touristique et possède à ce titre une Direction générale des devises Ouvrières à Ankara. De fait, avec l'extension du champ migratoire dès la fin des années soixante, la mise en place de politiques complexes de développement axées sur la rentabilisation de l'émigration durant les années soixante-dix (de Tapia 1996), puis les différents épisodes de déréglementation et de libéralisation économique déclenchés par Turgut Özal, président de la République, après avoir été ministre des Finances puis Premier Ministre, le transfert de devises est devenu libre et de nombreuses officines privées dans toutes les grandes villes et les régions touristiques brassent des sommes considérables sur des circuits commerciaux. Les Turcs s'y rendent pour transformer leurs retraites et salaires en devises fortes (US \$ et DM) avant qu'ils ne fondent devant l'inflation ${ }^{15}$.

133 L'actuelle Direction Générale des Devises Ouvrières (Isçi Dövizleri Genel Müdürlügü) est un service de 120 personnes qui édite, entre autres documents, un guide des services à l'usage des émigrés et des ressortissants turcs de retour $^{16}$. Ce document qui rappelle le 
guide édité par YIHGM est construit sur un modèle qui rappelle le guide OMI- Classeur français, à partir de fiches techniques amovibles permettant la réactualisation en fonction de l'évolution de la réglementation Nous avons pu utiliser les versions éditées entre 1989 et 2000. Il se divise en quatre chapitres :

134 - Services proposés par la Banque Centrale de Turquie (TCMB) : lettres de crédits, transferts de devises étrangères, régime d'importations dédouanées, réseaux TCMB dans le monde

135 - Services proposés par d'autres banques turques en relations avec des banques étrangères : lettres de crédit, comptes en devises, investissements en Turquie dans les domaines de l'agriculture, l'artisanat, l'immobilier, l'industrie, les fonds européens de développement...

136 - Services proposés par d'autres ministères ou administrations compétents en matière de migration internationale: Défense - le service militaire -, Éducation Nationale, Intérieur - la délivrance des passeports -, Finances et Douanes, Travail et Sécurité Sociale, Industrie et Commerce, Habitat, Déréglementation et Privatisation, réseaux administratifs turcs dans le monde - ambassades et consulats, attachés spécialisés...

137 - Autres services : PTT et mandats postaux, entreprises turques en Libye, obligations, marché de l'or, assurances automobiles gérées par le Touring Club de Turquie.

Le ministère des Transports et des Communications

138 Ce ministère (http://www.mt.gov.tr) n'a pas de compétence particulière en matière de migrations, ni internes, ni internationales. Par contre, parmi ses missions, celle du contrôle des activités de transports de passagers et indirectement de marchandises, voire l'encadrement des activités d'information et de communication (vidéocommunications et radio-télévisions, télématique et téléphonie...), sont de nature à intéresser les études sur les migrations par des activités de soutien et de logistique des flux migratoires. Ces sources indirectes, nous les avons bien souvent utilisées pour la définition et l'approche de la circulation migratoire, ou celle de la migration turque de transit à travers les Balkans et l'Europe centrale. De fait, existent pour le chercheur deux possibilités :

139 - la consultation des dossiers au ministère, éditeur directement ou par certains établissements publics rattachés de rapports et de statistiques,

140 - le recours direct aux sociétés, le plus souvent privées, opératrices dans le domaine concerné, avec en Turquie un avantage évident, celui de l'existence de compagnies consulaires et d'associations professionnelles spécialisées et plutôt efficaces (voir cidessous).

141 Une partie de ces études est d'ailleurs reversée au fond D.I.E. qui édite aujourd'hui plusieurs fascicules spécialisés (commerce extérieur, commerce extérieur par modes de transport, radio-télévision, transport maritime, transport aérien...).

142 Pour notre objet, on pourra se reporter à :

143 - statistiques des PTT et rapports d'activité,

144 - rapports d'activité de Türk Telekom S.A.,

145 - rapports d'activité et statistiques ferroviaires TCDD,

146 - rapports d'activité et statistiques aériennes (THY, IHY, OHY et autres compagnies de l'aviation civile, IHESI 1999), 
147 - rapports d'activité et statistiques routières de transport de passagers (compagnies d'autocars actives sur les lignes extérieures: Balkans, Europe occidentale, Caucase, Russie, Moyen-Orient),

148 - rapports d'activité et statistiques routières de transport de marchandises (sociétés de transport international routier, T.I.R. selon la Convention de New York de 1975, sur les destinations extérieures, dans les mêmes régions que les autocars),

149 - rapports d'activité et statistiques de transport maritime, passagers et marchandises (opérateurs publics ou en voie de privatisation comme Denizbank et TDI, opérateurs privés, armateurs).

150 Ce sont ces statistiques que nous pouvons considérer comme faisant partie de la logistique de l'émigration et en tous cas comme support de la circulation migratoire, étant entendu qu'il reste souvent difficile de déterminer la part des flux liés à la migration face à tous les flux intéressant un pays de plus en plus ouvert sur l'étranger, qu'il s'agisse de commerce extérieur ou de tourisme international. Dans le cas des compagnies aériennes nationales comme THY - Turkish Airlines, la distinction est faite entre vols "normaux ", charters émigrés et charters de pèlerins à destination de la Mecque et Djeddah. Cette distinction a cependant pour toutes les compagnies de moins en moins lieu d'être, devant la multiplication de tarifications spéciales. Dans le cas du transport routier de passagers, la même remarque s'impose, mais l'étude des lignes et des flux reste pourtant intéressante et renseigne sur les relations privilégiées des Turcs avec leur environnement régional (vie relationnelle des familles turques d'origine balkaniques, commerce à la valise, desserte de chantiers de travaux publics à l'étranger, desserte du champ migratoire).

Les statistiques annuelles des Postes

151 Depuis 1995, date de la création de Türk Telekom AS, les Postes éditent une série statistique recentrée sur leurs activités propres. Les chiffres disponibles chaque année permettent de mesurer les relations avec l'étranger (lettres, imprimés, colis et recommandés). Avant 1995, il était également possible de mesurer ces relations téléphoniques qui relèvent maintenant de la nouvelle agence.

152 Pour notre objet, il peut être utile de consulter les séries suivantes :

153 - Mandats postaux admis en Turquie par pays d'émission

154 - Mandats postaux expédiés de Turquie par pays de réception

155 - Courriers express reçus et expédiés par pays

156 - Trafic télégraphique international par pays (entrées et sorties)

157 Ces indicateurs fiables nous semblent pourtant de moins en moins pertinents pour les raisons suivantes :

158 - Les envois d'argent passent de plus en plus par le secteur bancaire qui dispose d'outils télématiques et informatiques perfectionnés.

159 - Le téléphone (et encore assez rarement le réseau Internet) se généralisent: les utilisateurs d'Internet sont parmi les principaux rédacteurs de courriers; les travailleurs sont pratiquement tous équipés de téléphones.

160 - Le trafic télégraphique passera bientôt au rang de curiosité historique: en 1989, 182000 télégrammes ont été échangés, on n'en compte plus que 23000 en 1998.

Statistiques des transports 
161 Ces statistiques sont nombreuses et détaillées, mais elles sont souvent disponibles dans les annuaires de DIE (cf. 1.2 1.) ou accessibles plus directement auprès des entreprises nationales ou privées, souvent regroupées en associations professionnelles (cf. 2). Si elles n'intéressent pas spécifiquement l'émigration, elles sont riches d'informations sur la logistique et le fonctionnement des acteurs de la circulation migratoire. En ce sens, il pourrait être utile de revenir plus précisément sur les «techniques » d'approche et d'évaluation de cette notion, dans la mesure où le cas turc s'y prête finalement assez bien.

Le ministère du Tourisme / T.C. Turizm Bakanlïgï

162 Ce ministère publie des séries statistiques depuis 1972. Les fascicules sont organisés selon une double logique, celle de l'accueil des touristes étrangers (tourisme international), celle des entrées-sorties de nationaux. Pour ce qui nous concerne ici, plusieurs tableaux devront être consultés :

163 - départs par année et mois,

164 - départs par départements de sorties (postes de douane) et mois,

165 - départs par départements de sorties (postes de douane) et modes de transport (route, fer, air, mer),

166 - départs par mois et modes de transport.

167 Les mêmes rubriques sont reprises pour l'entrée des nationaux sur le territoire turc. On dispose donc de huit séries de tableaux sur les passages de frontières. Ces statistiques sont en fait équivalentes de celles publiées par DIE chaque année.

168 Sur le site du ministère est aujourd'hui hébergé celui de l'Union turque des Agences de Voyage TURSAB (http://www.tursab.org) qui édite des statistiques qui peuvent venir en appui d'une réflexion sur les migrations, comme celles concernant le pèlerinage de la Mecque, les sorties de ressortissants turcs (déjà disponibles à DIE) ou le commerce à la valise (bavul ticareti).

Sources socio-professionnelles : compagnies consulaires, associations patronales des opérateurs de transport

169 Bien que non administratives, ces sources appartiennent généralement au domaine public par la structure administrative des établissements qui les éditent. Il peut s'agir de compagnies consulaires (Chambres de Commerce et d'Industrie, Chambres professionnelles spécialisées) ou d'associations de droit privé, mais fonctionnant comme des services publics (associations professionnelles). Nous les avons déjà évoquées par le biais du ministère des Transports et de la Communication qui collecte nombre de données de ce type pour les traiter de manière globale et contrôler les activités économiques afférentes.

170 Il convient cependant de présenter quelques unes de ces sources qui peuvent se révéler utiles pour les études des flux migratoires. Nous nous concentrerons sur le transport maritime et le transport routier de marchandises, avec les sources suivantes :

171 - les rapports DTO (Deniz Ticaret Odasi) : Chambre de Commerce Maritime d'Istanbul,

172 - les rapports d'activité de l'UND (Uluslararasï Nakliyeciler Dernegi): Association des Transporteurs Internationaux,

173 - le Guide du Transport (Nakliye Elkitabi) : nouveau document professionnel en vente libre,

174 - les rapports d'activité des compagnies aériennes (THY, IHY, OHY...), 
175 - les rapports d'activité et publications des organisations patronales (DEIK, TOBB, Chambres de Commerce et d'Industrie...).

Les rapports du secteur maritime (Deniz Sektörü Raporu)

176 Ces rapports annuels sont l'œuvre de la Chambre de Commerce Maritime d'Istanbul, compétente sur toute la Turquie, malgré quelques polémiques et quelques tentatives de scissions. Pour notre objet, peu d'informations concernent la migration, mais cette source nous intéresse pour la définition de la circulation migratoire ou encore l'approche des migrations de transit en provenance de Turquie, en particulier depuis 1990, avec le déclenchement de la crise yougoslave et la transformation de l'URSS en CEI. Un chapitre peut donc être signalé : l'évolution des trafics maritimes de passagers et de véhicules sur car-ferries et ro-ro. De quatre en 1990, les ro-ro sont ainsi passés à vingt-cinq en 1998, mais les traversiers, malgré un relatif développement, n'intéressent qu'une partie de la clientèle potentielle. Alors que le nombre de navires en service passe de cinq à huit (compagnie nationale TDI), le nombre de passagers transportés tombe de 216000 à 160 500, le nombre de véhicules transportés de 33000 à 30000 (1991 à 1996). On fera remarquer que dans ce domaine, ce sont toujours les compagnies grecques qui tirent pourtant leur épingle du jeu, à l'exception du trafic $\operatorname{TIR}^{17}$ (DTO). Les compagnies privées turques de car-ferries n'ont pas encore pu réellement, pour diverses raisons, concurrencer les grecques, alors que le secteur des transports routiers de marchandises a su le faire.

Les rapports d'activité de l'UND (Uluslararasï Nakliyeciler Dernegi) : Association des Transporteurs Internationaux

Il est clair que le trafic de camions immatriculés en Turquie ne peut s'apparenter au flux migratoire; toutefois, en tant que véhicule stricto sensu de denrées alimentaires, biens et objets « culturels » (certains ustensiles de cuisine, certains articles textiles ou de mobilier) produits en Turquie et commercialisés en Europe sur les lieux de résidence des immigrés, il participe de la logistique de la circulation migratoire comme de celle de l'établissement de communautés ou groupes structurés de population turque dans les pays du champ migratoire. Avec, en 1998, 794 membres et 57973 véhicules (dont 28 915 remorques) pour 697588 tonnes de capacité de charge, l'UND a des compétences proches de l'AFTRI française (Association Française des Transporteurs Routiers Internationaux). Avec la remarque énoncée précédemment, à savoir qu'il est très difficile de démarquer ce qui ressort de la circulation migratoire et ce qui ressort du commerce extérieur dans les données disponibles en Turquie ou à l'étranger, les rapports annuels UND sont hautement instructifs sur la structuration de l'espace relationnel turc et sur la construction du champ migratoire.

Il y a lieu de décrire brièvement la fiche technique que produit chaque membre de l'Association et le traitement général présenté comme rapport d'activité du secteur.

La fiche technique comprend les rubriques suivantes: nom du représentant de la société, capital inscrit, parc véhicules, capacité de transport, pays d'exercice, bureaux en Turquie et bureaux à l'étranger. Les pays d'exercice et les coordonnées des bureaux à l'étranger, toujours précises puisqu'il s'agit d'un document professionnel (en partie consultable sur Internet) nous renseignent sur la structuration du champ d'activité des transporteurs, celui-ci se confondant étroitement avec le champ migratoire ; une piste de recherche se trouve de fait ouverte quant à la structure des circuits 
d'approvisionnement du commerce turc en Europe et plus généralement entre Europe occidentale et pays de la CEI.

180 Le traitement général du rapport d'activité annuel est intéressant à plusieurs titres :

181 - l'évolution des trafics aux postes frontières selon les modes de transport (route, roro),

182 - les routes empruntées avec l'évolution des quotas annuels par pays de destination ou traversés,

183 - depuis 1996, les entrées de véhicules turcs par pays de destination.

184 On peut donc suivre mois par mois, année par année, pays par pays, l'évolution des trafics turcs et étrangers, en provenance, à destination, en transit, de et par le territoire turc. On y apprend que la Turquie est en relation par la route avec l'Afghanistan (1997 et 1998) et la Mongolie (1998).

Le Guide du Transport (Nakliye Elkitabi)

185 Ce guide, publié par une société privée d'Istanbul sous les auspices de plusieurs organismes, UND, UTIKAD et IATA, apparaît en 1998 et se trouve en vente libre. C'est avant tout un document édité pour un usage professionnel, comme les publications de l'UND ou la revue UTA (publications Uzman, Istanbul). Il reprend pour un public plus large le rapport d'activité de l'UND - par ailleurs disponible sur Internet - mais développe des chapitres et des informations sur des prestataires qui n'apparaissent pas dans les publications UND et surtout fait le tour de la quasi totalité de la branche d'activité : entreposage, transport routier intérieur (association TND), messagerie, constructeurs et équipementiers automobiles (associations OSD et TAYSAD), commissionnaires et agents (UTIKAD), transporteurs aériens (IATA - Turquie), armateurs (DTO), transport ferroviaire (TCDD) ... C'est donc toute la branche des transports de marchandises qui apparaît ici très organisée et qui peut à tout moment être démarchée pour une meilleure connaissance des relations de la Turquie avec son environnement économique.

Les compagnies aériennes

186 Depuis la Loi 2920 portant sur la déréglementation des transports aériens, cette activité a singulièrement été développée à la fois pour répondre aux besoins de la circulation migratoire (de Tapia 1996, MIGRINTER 1997-1999) et du tourisme international. Chaque compagnie édite statutairement un rapport d'activité annuel remis au ministère des Transports. DIE édite une nouvelle série de fascicules intitulés «Statistiques du transport Aérien ».

187 Ces statistiques peuvent être comparées à celles de l'OACI (trafic par étapes) analysées par Franck Gosselin dans le cadre du rapport IHESI (1999). Il en ressort deux faits majeurs :

188 - le développement de l'activité en Turquie ; on passe d'une compagnie nationale à 22 compagnies en 1996 (29 en 1993) possédant 110 appareils travaillant sur lignes exclusivement internationales (33 avions) ou alternativement intérieures et internationales (77 avions). THY sort renforcée malgré la concurrence d'Istanbul Airlines (IHY) et Onur Air (OHY).

189 - L'extension des dessertes internationales qui passent de la relation privilégiée, presque exclusive avec l'Europe occidentale, pour aboutir à l'implantation sur des destinations en Europe orientale, Russie, Caucase, Asie Centrale, Japon, Chine. Des 
essais sont faits vers Kazan (Tatarie), Simféropol (Crimée) ; le maintien ou l'abandon (Kazan) de ces lignes témoigne de conditions de rentabilité suffisantes ou non.

- La Turquie devient à son tour un pays plaque tournante du trafic aérien international. THY associée dans le groupe Qualiflyer (Swissair, Sabena, Austrian Airlines, Air Portugal, AOM) multiplie les accords bilatéraux avec les prestataires centrasiatiques et balkaniques (Azerbaycan Hava Yollarï, Uzbekistan Hava Yollarï, Air Kazakstan...) à partir du « hub » de Yesilköy (Aéroport Atatürk). Au moins 130 compagnies charter russes ou exsoviétiques ont un bureau de liaison en Turquie (Nakliye Elkitabï 2, 1998).

Les rapports UND ou les publications communes UND-UTIKAD (Nakliye Elkitabï 1999) signalent une extension croissante du champ couvert par les transporteurs routiers ou aériens, Turkish Airlines comprise. En particulier, l'apparition de bureaux de liaison des sociétés de transports TIR ou de points de vente THY à Moscou, Rostov na Donu, Krasnodar, Tachkent, Tirana ou Kazan, vient confirmer l'extraordinaire ouverture de l'économie turque sur l'étranger et la multiplication de colonies émigrées dont la logique première peut sembler fort différente de la migration de travail vers l'Europe (1958-1973), mais qui en réalité nous fait penser à une évolution du potentiel migratoire sous l'effet des progrès de l'éducation et de la formation (en Turquie) comme de la transformation de l'économie internationale.

Les rapports des associations patronales (DEIK, TOBB, TMB...)

De nombreuses organisations patronales, à l'instar de ce qui a été présenté ci-dessus pour les transporteurs, peuvent publier des données ou des analyses intéressant notre objet. Regroupées au sein d'une Union des Chambres et Bourses de Commerce et d'Industrie ayant son siège à Ankara, les Chambres de Commerce et d'Industrie publient de nombreuses études localisées ou thématiques. Toucheront notre thématique propre les rapports et études concernant particulièrement les investissements turcs à l'étranger, les chantiers du bâtiment et travaux publics. Ainsi l'Union d'Ankara (TOBB - Türkiye Odalar ve Borsalar Birligi), l'Institut des Relations Économiques extérieures (DEIK - Dïs Ekonomik Iliskiler Kurulu, http://www.deik.org.tr), parfois les CCI les plus puissantes, comme la Chambre de Commerce d'Istanbul (ITO Istanbul Ticaret Odasi), l'Union des Entrepreneurs du BTP de Turquie (TMB - Türkiye Müteahhitler Birligi) proposent rapports, études, guides pratiques à l'usage des investisseurs, susceptibles d'intéresser les chercheurs sur l'émigration. Cet intérêt réside dans deux aspects principaux : l'analyse de la pensée des acteurs économiques turcs, la production de statistiques partielles sur la présence turque à l'étranger, au moins sur deux types d'emplois, indépendants ou cadres d'entreprises turques installées à l'étranger, effectifs de travailleurs sur les chantiers ${ }^{18}$. Ces données qui restent parcellaires ont l'intérêt de nous renseigner sur les tendances nouvelles sur de nouveaux territoires d'immigration (Europe orientale et centrale, dont balkanique, Russie et Asie Centrale).

Le secteur bancaire : son rôle dans le champ migratoire, une source méconnue de données

193 Il s'agissait d'abord de banques étatiques dont la mission - le soutien à certaines activités de production et de services - et les compétences les amenaient à proposer des actions en faveur des travailleurs émigrés, les accompagnant pendant la migration ou les aidant à se réinsérer dans le tissu économique turc. Depuis plusieurs années, certaines banques privées s'étaient également intéressée au champ migratoire. Aujourd'hui, déréglementation, libéralisation, privatisation, sur un fond de crise 
financière chronique, sont venues singulièrement compliquer le tableau. Il n'en reste pas moins qu'au-delà du montant des remises des migrants ou des avoirs de ces mêmes migrants dans les pays d'accueil, les données bancaires semblent largement sousutilisées.

Les banques privées qui participent de la gestion des fonds des familles immigrées se trouvaient souvent alliées au secteur public. Pendant longtemps, la Turquie a vu coexister public et privé dans un vaste secteur d'économie mixte touchant aussi bien industries que finances (de Tapia 1996b). Il est clair que l'actuelle crise financière, faisant suite à plusieurs vagues mal assurées de privatisations - où nombre de banques publiques privatisées ont subi des pertes considérables - remettra en cause tout l'édifice à la fois en Turquie et dans les pays du champ migratoire. Il est bien trop tôt pour émettre des conclusions définitives, aussi préférons-nous ici nous fonder sur la situation antérieure de l'année 1997 qui permettra de montrer la complexité du réseau bancaire qui a drainé le champ migratoire. Nous n'avons que peu, au-delà de la recherche personnelle de doctorat de géographie, abordé ce secteur complexe, mais il a toujours été pris en compte dans la définition des réseaux transnationaux.

Dans les guides édités par le ministère du Travail et de la Sécurité Sociale (YIHGM 1991) et la Banque Centrale de Turquie (TCMB 1989 à 2000) sont décrites les modalités de transfert suivantes; en gardant l'année 1997 comme référence :

Les possibilités d'ouvertures de compte bancaires et comptes épargne

- Comptes en devises (Süper Döviz Hesabi, niveau minimum requis en 1997 : 15000 US\$ ou 30000 DM) auprès de la Banque Centrale de Turquie (TCMB), accords avec les Dresdner et Bremer Bank AG, Algemeine Bank Nederland BV sur ouverture de livrets d'épargne.

- Aux USA, les banques suivantes ont des accords avec la Turquie pour les transferts de fonds : American Express, American Security, Banca Commerciale Italiana, Bank of New York, Bankers Trust $\mathrm{C}^{\circ}$, Chase Manhattan Bank, Chemical Bank, First National Bank (Boston, Chicago), Manufacturers Hanover Trust $\mathrm{C}^{\circ}$, Morgan Guaranty, Riggs National Bank, TC Ziraat Bankasï New York (Crédit Agricole turc).

198 - Comptes de dépôt sur lettre de crédit en devises, niveau minimum requis en 1989 : 2500 \$ ou 15000 francs (français). auprès de la Banque Centrale de Turquie (TCMB), accords avec les Dresdner et Bremer Bank AG, Algemeine Bank Nederland BV sur ouverture de livrets d'épargne.

199 - Comptes de dépôt en devises ou TL auprès des autres banques turques, publiques ou privées.

200 Les Banques turques suivantes avaient en 1997 des succursales dans les pays du champ migratoire : TC Ziraat Bankasï (Crédit Agricole ; publique), Türk Dïs Ticaret, Türkiye Emlak Bankasï (Crédit Immobilier, publique), Türkiye Garanti Bankasï (privée), Türkiye Halk Bankasï (Banque populaire, publique, artisanat, commerce, PME), Türkiye Imar Bankasi (immobilier), Türkiye Is Bankasï (publique, investissement industriel), TCMB (Banque Centrale), Akbank (privée), Etibank (secteur minier, investissement, étatique aujourd'hui privatisée), Iktisat Bankasï (privée), Pamukbank (privée), Sekerbank (mixte, les planteurs de betteraves sucrières), TÖBANK (Banque mutuelle des Enseignants), Türk Ticaret Bankasï, Tütünbank (mixte, les planteurs de tabac), Türkiye Vakiflar Bankasi (publique, les Fondations Religieuses), Yapi ve Kredi Bankasï (publique, immobilier). Au total, 103 succursales de banques turques fonctionnent dans les pays du champ migratoire. La TCMB a quatre centres administratifs en Allemagne, aux Etats-Unis et au Royaume-Uni. 
S'ajoutent banques agréées et mixtes (Banque du Bosphore en France, Demir-Halk Bank aux Pays-Bas, Turkish Bank UK Ltd à Londres).

En France, les intérêts turcs sont représentés par la Banque du Bosphore, filiale de Emlak Bankasï, la Banque Internationale de Commerce appartenant à Erol Aksoy, homme d'affaires stambouliote; des accords existent avec la Société Générale, la Banque Monte Paschi, le Crédit Lyonnais, la BNP ou la Caisse d'Épargne.

Les transferts de marchandises importables par les émigrés résidents à l'étranger

L'administration publie chaque année des listes complètes de véhicules, outils, machines... à usage professionnel et de matériels, meubles, biens de consommation à usage familial et privé, cadeaux dédouanés ou partiellement dédouanés. Peuvent être importés en 1997 à usage professionnel: machines à écrire et ordinateurs, postes de soudure, tronçonneuses et scies mécaniques, trayeuses et barattes électriques, fax et quelques machines pour les ateliers mécaniques. Cette liste est considérablement limitée par rapport aux années précédentes. En 1989, elle comprenait: machines électriques de coupe, chaudières, pompes et centrifugeuses, générateurs, turbines (vapeur, gaz, électricité), machines agricoles, machines de chantier, équipements de laboratoires, équipements médicaux et toute une série de véhicules, y compris autocars à deux étages et hélicoptères ! En tout, la liste de 1989 comprenait 176 rubriques.

L'administration publie aussi chaque année des listes des importations entrant dans le cadre de dons à des collectivités locales ou à des œuvres sociales. En 1997, la liste comprend: automobiles VL, $4 \times 4$, taxis, camionnettes, minibus (moins de 16 places), autobus, camions, tracteurs routiers, de moins de 5 ans, souvent envoyés par les migrants aux municipalités, à des services locaux de police, des hôpitaux, des écoles. S'ajoutent des véhicules spécialisés qui ne doivent pas dépasser 10 ans: ambulances, véhicules-incendie, tracteurs, machines de chantier, véhicules et camions de salubrité publique (benne ou camion spécial de ramassage d'ordures ménagères), corbillards, bibliobus... Ces dons sont assez nombreux et s'apparentent aux dons islamiques traditionnels qui consistaient, par exemple à construire une fontaine en ville ou au bord d'une route (sebil).

Les aides et crédits à la création d'entreprise et les crédits immobiliers

Une banque spéciale, DESIYAB (Banque Étatique pour les Investissements Industriels des Travailleurs) avait été créée dans les années soixante-dix. Elle a depuis fusionné avec la Banque du Tourisme (publique) pour devenir la Banque de Développement de Turquie (TKB - Türkiye Kalkinma Bankasi). Elle offre ses services aux émigrés pour la création d'entreprises industrielles et touristiques. La Banque Populaire (Halkbank ou Türkiye Halk Bankasi) est mandatée depuis longtemps pour accompagner les émigrés de retour lors de la création d'entreprises artisanales.

205 • Crédits à la création d'entreprise sur fonds germano-turc KSF 1 gérés par la Banque Populaire,

206 - Fonds d'incitation du Kreditanstalt für Wiederaufbau (KFW) pour PME en zones éligibles,

207 • Crédits KÖF II pour les sociétés ouvrières créées au départ par des actionnaires émigrés,

208 - projets éligibles sur les zones prioritaires au développement, zones industrielles et zones franches (Halkbank, Türkiye Kalkïnma Bankasi).

209 Les zones prioritaires sont chaque année définies par l'Administration (DPT = DATAR turque) depuis les années soixante. Il peut s'agir de départements ou d'arrondissements 
considérés comme sous-développés, susceptibles d'être développés et à ce titre éligibles pour diverses catégories d'aides (remises d'impôts, incitations financières, crédits d'équipement, à l'importation, dédouanement...). Les investissements en zone industrielle organisée (OSB - Organize Sanayi Bölgesi), en zone artisanale (KSS - Küçük Sanayi Sitesi), en zone franche (Serbest Bölgeler) sont de même susceptibles d'être aidés par l'Etat, au moyen de licences d'encouragement (tesvik belgesi). La réglementation est en ce domaine particulièrement complexe. Les émigrés font le plus souvent partie des catégories d'investisseurs éligibles du fait même de leur qualité de migrant.

210 - Crédits au commerce et aux activités touristiques gérés par Halkbank, Türkiye Kalkïnma Bankasi,

211 • Crédits ouverts par les banques d'État spécialisées (Crédit Agricole, Crédit Industriel...) ou la Garanti Bankasï privée à partir de leurs agences européennes ou autres sur le champ migratoire (USA, Australie, pays arabes, etc.).

212 - Comptes épargne logement ouverts par les Caisses d'Épargne Allemandes pour construction de logements en Turquie plafonnés à 100000 DM par logement. Les Sparkassen allemandes comme la Caisse d'Épargne française collaborent avec Emlak Bankasï.

213 Toutes ces organisations bancaires, pour certaines d'entre elles très présentes dans les pays du champ migratoire - la France fait plutôt figure d'exception par sa faible implantation bancaire turque - éditent bien évidemment des rapports d'activité. L'étude de ces rapports peut être renforcée au niveau local par les dossiers remis par les entreprises aux administrations départementales. Les études REMPLOD ou Isoplan sur les entreprises créées par des émigrés et sur le contexte régional montrent la richesse de ces sources La liste des implantations et des accords de partenariat avec des banques de pays d'immigration ou de transit peut jouer là aussi le rôle d'indicateur sur une éventuelle présence allant vers la stabilisation de migrants, ce qui semble bien être le cas dans toute l'Europe où les pays de transit semblent presque partout se transformer en pays de résidence. On pourra là aussi comparer avec les activités des banques locales en relation avec la Turquie. Une augmentation subite de transferts vers la Turquie ou en provenance de Turquie peut indiquer la présence de réseaux en relation avec ce pays ${ }^{19}$.

Conclusion

214 Les statistiques turques sont riches d'enseignements et relativement faciles d'accès. Être turcophone est sans doute un avantage, mais ce n'est pas une condition nécessaire: les statistiques sont souvent proposées en anglais (mais la «littérature grise » n'est généralement disponible qu'en turc). Dans l'ensemble, les données apparaissent relativement fiables et relèvent de la tradition ottomane qui comptait et décomptait les aspects les plus divers de la vie sociale et économique dans un évident souci de contrôle des populations, contrôle militaire et fiscal permettant à l'empire de maintenir sa place dans le concert des nations. Les Turcs sont les premiers à dénoncer les insuffisances de leur appareil statistique ou les détournements de procédures, par exemple des exportations fictives. Les statistiques sur l'emploi sont souvent sujettes à caution dans la mesure où l'activité économique reste très fluctuante, avec un secteur informel très important. En ce qui nous concerne ici, les statistiques de passage de frontière sont probablement parmi les plus fiables, avec des frontières bien gardées, à l'exclusion du quart sud-est du pays, où cependant les militaires se déplacent vite en cas de besoin (afflux kurdes de 1988 et 1991). 
215 Entre le pays d'origine et les pays d'immigration d'Europe occidentale, il serait utile d'examiner systématiquement les passages de frontières par nationalité, ressortissants turcs et populations en rapport avec le territoire turc pour avoir une idée des flux véritables passant d'un compartiment à l'autre. Nous avons une idée nette des flux sur les frontières turques, même si des passages clandestins sont toujours possibles. Nous avons une idée nette des présences turques et assimilées (nationaux et naturalisés) dans les pays traditionnels d'immigration, Allemagne, France, Belgique, moins pour les nouveaux pays d'immigration, de la Finlande au Kazakhstan. L'Italie doit être cernée de plus près dans ce domaine, en ce qui concerne la population d'origine turque qui semble en augmentation sensible. Par contre, dans tous les pays d'Europe orientale, l'immigration est un fait nouveau pour des législations et des réglementations en construction. Il serait donc nécessaire au préalable de se livrer dans tous ces Etats au même exercice qu'en Turquie, de façon à comparer les flux sur les frontières nationales avec les mêmes flux sur les étapes intermédiaires, Bulgarie, Roumanie, Yougoslavie, Macédoine, Grèce, Albanie, Hongrie, Ukraine, Russie, Biélorussie, Estonie, Lettonie, Lituanie, Pologne, Finlande.

Nous avons essayé ici de rendre compte de l'ensemble de l'appareil statistique turc susceptible d'être utilisé pour une meilleure connaissance de la totalité des aspects de la présence turque en Europe et dans le monde par le biais de la mobilité et de la migration internationale. Cet appareil qui décrit des formes très variées de mobilité des hommes, des marchandises, de l'information et des capitaux permet de cerner et de mieux définir des réseaux de natures très différentes. C'est à n'en pas douter dans l'analyse fine de ces catégories statistiques et dans la comparaison entre flux d'essences différentes (hommes, biens matériels, flux immatériels) qu'il est possible de trouver les indicateurs de mouvements non apparents dans les statistiques européennes ou nationales telles qu'elles existent (SOPEMI, Eurostat, UNHCR...). Restent à confronter résultats acquis en Turquie, pays de départ, dans les pays d'immigration et dans les pays de transit, bien que ce terme, légitime lorsque les pays de l'Europe orientale ne participait pas directement du champ migratoire turc, semble ne plus être d'actualité.

\section{BIBLIOGRAPHIE}

ABADAN Nermin (1965), Batï Almanya'daki Türk Isçileri ve Sorunlarï, Ankara, Devlet Planlâma Teskilâti.

ABADAN-UNAT Nermin \& Nese KEMIKSIZ (1986), Türk Dïs Göçü 1960-1984, Yorumlu Bibliografya, Ankara \& Bonn, Faculté des Sciences Politiques de l'Université d'Ankara, Zentrum für Türkeistudien.

ABADAN-UNAT Nermin (1993), Turkey : Late Entrant into Europe's Work Force, in D. KUBAT Ed., The Politics of Migration Politics, pp. 307-336.

ANDREWS Peter A. et alii (1989), Ethnic Groups in the Republic of Turkey, L. Reichert Verlag, Beihefte für Tübinger Atlas des Vorderes Orients B 60. 
BALCI Bayram Ed. \& Bertrand BUCHWALTER Collab. (2000), La Turquie en Asie Centrale. La conversion au réalisme. Les Dossiers de l'IFEA (Série la Turquie aujourd'hui 5), Istanbul, IFEA.

BOOS-NÜNNING Ursula (1989), Ed., Die türkische Migration in deutschsprachigen Büchern 1961-1984, Opladen, Zentrum für Türkeistudien.

DAGM - T.C. Basbakanlïk Devlet Arsivleri Genel Müdürlügü (1993), Türkiye Dïsïndaki Türk Vatandaslarï Bibliografyasi - A Bibliography of Turkish Citizens out of Turkey, Ankara.

DIE - T.C. Devlet Istatistik Enstitüsü (annuel), Türkiye Istatistik Yilligi - Statistical Yearbook of Turkey, Ankara, voir aussi : http://www.die.gov.tr

DIE -T.C. Devlet Istatistik Enstitüsü (1994, 1999), Turizm Istatistikleri 1990, 1991 et 1995 [Statistiques du Tourisme], Ankara, T.C. Basbakanlïgï.

DIE - T.C. Devlet Istatistik Enstitüsü (1996) Genel Nüfus Sayïmïna göre Iç Göçler ve Göç Eden Nüfusun Sosyal ve Ekonomik Nitelikleri [Migrations Internes et Caractéristiques Socio-économiques de la Population migrante au Recensement Général de la Population de 1990], Ankara, T.C. Basbakanlïgï.

DIE - T.C. Devlet Istatistik Enstitüsü (annuel), Dïs Ticaret Yillik Istatistikleri [Annuaire Statistique du Commerce Extérieur], Ankara, T.C. Basbakanlïgï.

DIE - T.C. Devlet Istatistik Enstitüsü (1988 à 1992), Deniz Tasitlarï Istatistikleri [Statistics of Sea Vessels], Ankara, T.C. Basbakanlïii.

DIE - T.C. Devlet Istatistik Enstitüsü (1988-1994), Kabotaj ve Uluslararasï Deniz Tasïmasï Istatistikleri [Statistics on Coastal and International Sea Transportation], Ankara, T.C. Basbakanlïgï.

DIE - T.C. Devlet Istatistik Enstitüsü (1990-1993), Motorlu Kara Tasïtlarï Istatistikleri [Statistiques des Véhicules à Moteur], Ankara, T.C. Basbakanlïgï.

DIE - T.C. Devlet Istatistik Enstitüsü (1993 \& 1994), Ulastïrma Istatistikleri Özeti 1991, 1992, 1993 [Abrégé des Statistiques des Transport], Ankara, T.C. Basbakanlïgï.

DPT - T.C. Devlet Planlama Teskilâtï (1990), Havayolu Ulastïrmasï [Le Transport Aérien], DPT - T.C. Devlet Planlama Teskilâtï, Bes Yíllïk Kalkïnma Planï, ÖIK Raporu, DPT 2217, ÖIK 361, Ankara.

DPT - T.C. Devlet Planlama Teskilâtï (1982), 1983-1993 Ulastïrma Ana Planï [Plan Général des Transports, 1973-1993], Ankara, T.C. Basbakanlïgï.

DPT - T.C. Devlet Planlama Teskilâtï (1991), Demiryolu Ulastïrmasï : Özel Ihtisas Komisyonu Raporu [Le Transport Ferroviaire], Ankara, T.C. Basbakanligï, DPT 2275.

DPT - T.C. Devlet Planlama Teskilâtï (1992), Karayolu Ulastïrmasï [Le Transport Routier], Ankara, T.C. Basbakanlïgï, DPT 2306.

DPT - T.C. Devlet Planlama Teskilâti (1995), 1995 Yïlï Geçis Programï Destek Çalïsmalarï, Ekonomik ve Sosyal Sektörlerdeki Gelismeler [Développements des secteurs économiques et sociaux, travaux de soutien au programme de transition de 1995], Ankara, DPT.

DPT - T.C. Devlet Planlama Teskilâtï (1995), 1995 Yilï Geçis Programï [Programme de Transition pour 1995], Ankara, DPT.

DTO - Deniz Ticaret Odasï, Turkey Shipping Handbook (1987), Istanbul ve Marmara Ege, Akdeniz, Karadeniz Bölgeleri Deniz Ticaret Odasï.

DTO - Deniz Ticaret Odasï (Annuels), Deniz Sektörü Raporu [Rapport sur le Secteur Maritime], Istanbul ve Marmara Ege, Akdeniz, Karadeniz Bölgeleri Deniz Ticaret Odasï, Istanbul. 
DTO - Deniz Ticaret Odasï (1996), Turkey Shipping Handbook 1996, Istanbul ve Marmara Ege, Akdeniz, Karadeniz Bölgeleri Deniz Ticaret Odasï, Istanbul.

ELMAS Hasan B. (1998), Turquie-Europe, une relation ambiguë, Syllepse, Paris.

EUROSTAT (1992), Bevölkerungsstatistik / Demographic Statistics / Statistiques Démographiques, Luxembourg, Office des Publications Officielles des Communautés Européennes.

GITMEZ Ali S.(1983), Yurtdïsina Isçi Göçü ve Geri Dönüsler [Emigration et Retours] Istanbul, Alan.

GÖKDERE Ahmet (1978), Yabancï Ülkelere Isgücü Akïmï ve Türk Ekonomisi Üzerine Etkileri [Le flux de main-d'œuvre vers l'étranger et ses effets sur l'économie turque], Ankara, Türkiye Is Bankasï.

ICAO/OACI, Traffic by Flight Stage / Trafic par étapes, Organisation de l'Aviation Civile Internationale, Montréal, Séries TF, 1982/1992.

ICDUYGU Ahmet (1995), Turks in Australia, in Stéphane de Tapia (Ed.), Turcs d'Europe... et d'ailleurs, Les Annales de l'Autre Islam, vol. 3, pp. 253-278.

ICDUYGU Ahmet (1995), Transit Migration in Turkey, Budapest, IOM.

ICDUYGU Ahmet (1996), Transit Migration in Turkey, Bogaziçi Journal : Review of Social, Economic and Administrative Studies, 10 (1-2), pp. 127-142.

ICDUYGU Ahmet (2000), Transit Migration in Turkey, Migration Newsletter 1, mars 2001, extrait d'International Social Science Journal de septembre 2000 (http://www.calisma.gov.tr : site du ministère du Travail et de la Sécurité Sociale).

IIBK - Is ve Isçi Bulma Kurumu [Office du travail et du Placement], Istatistik Yilligï, Statistical Yearbook, publication annuelle, voir aussi http://www.iskur.gov.tr

IHESI - Institut des Hautes Etudes sur la Sécurité Intérieure (1999), Programme ODYSSEUS: Étude méthodologique de l'immigration de transit et de rebond à partir d'un flux type, les Turcs. Paris, IHESI. Contributions de Samim Akgönül, Franck Gosselin, Yücel Gül, Stéphane de Tapia.

IOM / OIM - International Organization for Migration (1994-1995), Transit Migration in Bulgaria, Poland, Ukraine, Turkey, Budapest, IOM Migration Information Programme.

KARPAT Kemal H. (1995), The Turks in America, in Stéphane de Tapia (Ed.), Turcs d'Europe... et d'ailleurs, Les Annales de l'Autre Islam, vol. 3, pp. 231-252.

KRAMER Heinz (2000), A Changing Turkey : the Challenge to Europe and the United States, Washington Brookings.

MARTIN Philipp (1991), Bitmeyen Öykü : Batï Avrupa'ya Türk Isçi Göçü - The Unfinished Story, Bureau International du Travail, Genève, Ankara pour la version turque.

Migrations Études (1998), La circulation migratoire. Bilan des travaux, 84, Paris, ADRI.

Migrations Études (2000), Registres de populations, migrations internationales et dénombrement de la présence étrangère en Allemagne, 95, Paris, ADRI.

MIGRINTER (1997 \& 1999), La circulation migratoire dans les diasporas ouvrières de la Méditerranée, vol. 1: Turquie, vol. 2: Maroc, Poitiers et Poitiers-Strasbourg, rédigés par Stéphane de TAPIA, Mohammed CHAREF, avec la collaboration de Catherine GAUTHIER, Poitiers.

Nakliye El Kitabï - Transport Handbook (1998), Istanbul, Sagün.

RAPPER Gilles de (2000), Les Albanais à Istanbul, Les Dossiers de l'IFEA (série : la Turquie aujourd'hui), 3, Istanbul, IFEA. 
OCDE/SOPEMI (1980-1990), Rapport Annuel, Paris, O.C.D.E.

OCDE/SOPEMI (1992 sq.), Tendances des Migrations internationales, Rapports annuels, Paris, O.C.D.E. ONU - Organisation des Nations Unies (1988), Manuel T.I.R.. Convention Douanière relative au Transport International de Marchandises sous le couvert de Carnets T.I.R., Commission Economique pour l'Europe.

ONU - Organisation des Nations Unies (1993), Recensement de la Circulation Motorisée sur les Grandes Routes de Circulation Internationale (Europe), Commission Economique pour l'Europe, 10 livrets.

PTT - PTT Isletmesi G.M. (annuel), P.T.T. Faaliyet Raporu [Rapport d'Activité des P.T.T.], Ankara, Imprimerie des P.T.T.

PTT - PTT Isletmesi G.M. (annuel), P.T.T. Istatistikleri / Statistics, Ankara, Imprimerie des P.T.T. SCHWARZ Thomas \& ERSÖZ Ahmet (1990), Bibliographie zur Türkischen Migration, Berlin, Parabolis. SECCOMBE Ian .J. \& LAWLESS Richard. I (1986), Between Western Europe and the Middle East : Changing Patterns of Turkish Labor Migration, Revue Européenne des Migrations Internationales, 2 (1) , pp. 37-58.

SIMON Gildas (1995), Géodynamique des migrations internationales dans le monde, Paris, PUF

SEN Faruk, ULUSOY Yunus \& Güray ÖZ (1999), Avrupa Türkleri, Istanbul, Cumhuriyet.

TAPIA Stéphane de \& Gilles DUBUS (1995), Les migrations originaires de Turquie. Biliographie francophone. MIGRINTER, Poitiers, miméo.

TAPIA Stéphane de (1989), Emigrations, retour : mutations du champ migratoire turc, Revue de l’Orient Musulman Méditerranéen, 52/53, pp. 255-273.

TAPIA Stéphane de (1992), Le champ migratoire turc : évolutions et mutations (1974-1992), Peuples Méditerranéens, 60, pp. 135-152.

TAPIA Stéphane de (1995), L'émigration turque : des « portes de l'étranger » aux " portes d'entrée » (el kapilarindan giris kapilarina) ? Annales de l'Autre Islam, 3, pp. 27-44.

TAPIA Stéphane de (1996), Échanges, transports et communications : circulation et champs migratoires turcs, Revue Européenne des Migrations Internationales, 12 (2), pp. 45-71.

TAPIA Stéphane de (1996), L'impact régional en Turquie des investissements industriels des travailleurs émigrés, Paris, Istanbul, L'Harmattan - IFEA (Varia Turcica XIX).

TAPIA Stéphane de (2000), De l'héritage impérial aux migrations ouvrières, les Turcs d'Europe, in Daniel Balland Dir., Hommes et Terres d'Islam. Mélanges offerts à Xavier de Planhol, IFRI, Téhéran (Bibliothèque Iranienne 53, Tome 2), pp. 295-308.

TAPIA Stéphane de (2001), Rôle des flux migratoires et des réfugiés dans la construction d'un Etat, in Luc Cambrézy et Véronique Lassailly-Jacob, Populations réfugiées, de l'exil au retour, Paris, IRD Editions, pp. 119-148.

TAPIA Stéphane de (à paraître), Transnational Migration and Entrepreneurship of Migrants : Between Turkey, Europe and the Turkic World, in Christiane Harzig \& Danielle Juteau Eds., The Social Construction of Diversity, New York, Berghahn Books.

TCMB - Türkiye Cumhuriyeti Merkez Bankasï / Banque Centrale de Turquie, Yurt Dïsïnda « çalïsan Vatandaslarïmïza » çesitli Kurumlar Tarafindan Sunulan Hizmetler ve Açiklamasï [Services présentés par diverses institutions en faveur des travailleurs expatriés et commentaires], Ankara 1989-2000, révisions selon évolution des réglementations. 
THY - Türk Hava Yollarï / Turkish Airlines (annuel), THY Faaliyet Raporu [Rapport d'Activité de Turkish Airlines] Istanbul, voir aussi : http://www.turkishairlines.com

TIKA - Türk Isbirligi ve Kalkïnma Ajansï, Eurasian File (TIKA, Ankara, mensuel), voir aussi http:// www.tika.gov.tr

TIKA - Türk Isbirligi ve Kalkïnma Ajansï, Azerbaycan Ülke Raporu [Rapport sur l'Azerbaïdjan], Ankara, TICA Publication Department, 3, 1993 - 1994.

TIKA - Türk Isbirligi ve Kalkïnma Ajansï, Kazakistan Ülke Raporu [Rapport sur le Kazakhstan], Ankara, TICA Publication Department, 4, 1993 - 1994.

TIKA - Türk Isbirligi ve Kalkïnma Ajansï, Kïrgïzistan Ülke Raporu [Rapport sur la Kirghizie], Ankara, TICA Publication Department, 5, 1993 - 1994.

TIKA - Türk Isbirligi ve Kalkïnma Ajansï, Özbekistan Ülke Raporu [Rapport sur l'Ouzbékistan], TICA Publication Department, 6, Ankara, 1993 - 1994.

TIKA - Türk Isbirligi ve Kalkïnma Ajansï, Türkmenistan Ülke Raporu [Rapport sur le Turkménistan], TICA Publication Department, 7, Ankara, 1993 - 1994.

Türk Telekom A.S. (annuel), Faaliyet Raporu [Rapport d'activité], Ankara, voir aussi http:// www.telekom.gov.tr

T.C. Turizm Bakanlïgï / ministère du Tourisme (1994-1995), Turizm Istatistikleri Bülteni [Bulletin de Statistiques du Tourisme], TB Yatïrïmlar Genel Müdürlügü Arastïrma ve Degerlendirme Daire Baskanlïgï, Ankara, voir aussi http://www.tursab.org.tr

TOUMARKINE Alexandre (1995), Les migrations des populations musulmanes balkaniques en Anatolie (1876-1913), Istanbul, Isis.

T. C. Ulastïrma Bakanlïgï / ministère des Transports (1991), Ulastïrma ve Haberlesme 1983-1990 [Transport et Information, 1983-1990], Ankara, voir aussi http://www.mt.gov.tr

UATOD - Uluslararasï Anadolu ve Trakya Otobüsçüler Dernegi / Association des Autocaristes Internationaux de Thrace et d'Anatolie, rédigé par Tahir PEK'AR (1993), Karayoluyla Yolcu Tasïmaciligï ile Ilgili Genel Istatistik Bilgiler [Informations générales et statistiques relatives au transport international routier de voyageurs], Istanbul, 2, 1993, voir aussi http:// www.uatod.org.tr

UNBEHAUN Horst (1995), Migration professionnelle vers le Proche-Orient, la Russie, l'Asie Centrale, in Stéphane de Tapia (Ed.), Turcs d'Europe... et d'ailleurs, Les Annales de l'Autre Islam, 3, pp. 279-309.

UND - Uluslararasï Nakliyeciler Dernegi / International Transporters Association, Sektör Raporu / Annual Report (1989 à 1998), Istanbul, voir aussi UND'nin Sesi [la Voix de l'UND], revue mensuelle et http://www.und.org.tr

Y.I.H.G.M. - Yurtdïsï Isçiler Hizmetler Genel Müdürlügü / Direction Générale des services aux Travailleurs Expatriés (annuel), Yillik Raporu [rapport annuel de la Direction des Services aux Travailleurs Emigrés], Ankara, voir aussi : http://www.calisma.gov.tr

Y.I.H.G.M. - Yurtdïsï Isçiler Hizmetler Genel Müdürlügü / Direction Générale des services aux Travailleurs Expatriés (1991), Yurtdïsïnda çalïsan ve Yurda Dönen Vatandaslarïmïza Sunulan Hizmetler. Rehber [Guide des Services proposés aux émigrés à l'étranger et de retour], ministère du Travail et de la Sécurité Sociale, Ankara. 


\section{NOTES}

[F. En raison des problèmes de transfert de fontes de caractères entre modèles d'ordinateurs et équipements en polices d'écriture, la graphie latine turque n'a pas pu être respectée. Que les lecteurs turcophones veuillent bien nous en excuser.

1. -La bibliographie établie par Nermin Abadan-Unat avec l'assistance de Nese Kemiksiz (1986) rassemble 1154 titres dans toutes les langues, celle d'Ursula-Boos-Nünning (1990), 1190 titres, uniquement en allemand, celle de Thomas Schwarz et Ahmet Ersöz (1990), 2755 titres. Ce ne sont pas des cas isolés, la Direction Générale des Archives Nationales (DAGM - Devlet Arsivleri Genel Müdürlügü, 1993, Ankara) s'est essayée au même exercice (932 titres, toutes langues).

2. -Selon l'édition allemande de Milliyet (25.06.2001), 1700000 Turcs se seraient inscrits auprès de l'administration américaine pour bénéficier du tirage au sort lié à la greencard alors que le quota réservé aux ressortissants turcs ne serait que de 1000 places. Skylife, mensuel de la compagnie Turkish Airlines, fait d'ailleurs de la publicité pour cette forme d'émigration.

3. -L'ouvrage de Nermin Abadan, publié par Devlet Planlama Teskilatï en 1965, est le premier d'une très longue série d'études que mèneront chercheurs turcs et étrangers. Son auteur, aujourd'hui retraitée, a accompagné l'émigration turque sur toute son histoire, parallèlement à d'autres travaux reconnus sur la sociologie des femmes et de la famille en Turquie.

4. -A l'image de Yozgat Katalogu 1990 [Catalogue de Yozgat 1990], édité par l'Association Culturelle et de Solidarité des Originaires de Yozgat à Istanbul, en deux volumes, 645 pages et quelques (photos, publicités non paginées), présentant histoire, géographie, économie, culture, personnages célèbres, administrateurs, élus locaux ..., le tout introduit par deux ministres d'état (Cemil Çiçek et Ercüment Konukman), le ministre de l'Agriculture, de la Forêt et des Affaires Rurales (Lüftullah Kayalar), tous trois originaires du département, le préfet et le maire de Yozgat! Document prêté par un originaire de Yozgat vivant à Strasbourg.

5. -Anecdote souvent mise en avant par Gaye Salom ou Altan Gokalp lors de leurs premières interventions écrites ou orales sur l'immigration turque en France, le cas de travailleurs non francophones, peu scolarisés, qui mirent en difficulté des patrons indélicats devant les juridictions professionnelles parisiennes ; ils avaient accompagné la signature de leurs contrats de codicilles en turc signifiant « je signe sans comprendre ", ce qui prouva leur bonne foi.

6. -L'actuel ministère est le ministère du Tourisme (TC Turizm Bakanligi). Culture et Tourisme ont souvent été regroupés, mais en Turquie comme ailleurs, l'envergure des ministères est sujette à variation selon la composition des gouvernements et l'idée que l'on se fait du thème abordé.

7. -Peu connu en France, ce sujet est toutefois traité par de nombreux auteurs, dont quelques Français, dans ses dimensions historiques ou plus récentes. La bibliographie serait en fait fort longue. On citera parmi les auteurs français ou francophones, Alexandre Toumarkine, Paul Dumont, François Georgeon, Nedim Tarhan, Samim Akgönül ou Stéphane de Tapia pour l'une ou l'autre approche du phénomène. 8. -En 2000, pour les trois premiers mois de l'année, 23 Algériens, 75 Marocains et 16 Tunisiens avaient été arrêtés sur les frontières, généralement en Thrace orientale où se joignent les frontières turques, bulgares et grecques. Pour 1999, les chiffres sont respectivement de 228, 356, 60. Pour la période 1995-premier trimestre 2000, la police 
turque a appréhendé 579 Algériens, 900 Marocains, 252 Tunisiens. Des travaux universitaires sur la présence maghrébine à Istanbul sont en cours.

9. -Le reportage touristique de Patricia Daunt pour la revue anglo-américaine de Turquie, Cornuscopia, (12 - 2), 1997 : 54-73, met en relation l'architecture villageoise prestigieuse de la vallée de Hemshin avec l'émigration de pâtissiers et de restaurateurs vers la Russie du sud, le Caucase, la Crimée ou Téhéran. Bien d'autres indications peuvent être relevées chez des géographes (Necdet Sözer) ou des historiens (Kemal Karpat) de ces filières des originaires des vallées pontiques vers la Russie ou les pays riverains de la Mer Noire occidentale (Bulgarie, Roumanie). A notre connaissance, il n'existe cependant pas d'étude systématique de ces filières de gurbetçi (travailleurs vivant de la nostalgie ; ce sera aussi le terme employé pour les migrants d'Allemagne. De fait, les modifications successives des frontières de l'empire ottoman ont bien souvent transformé en migration internationale ce qui était une migration de travail interne.

10. -Là où les statistiques européennes distingueraient « réfugiés de souche turque » (= Aussiedler) et « réfugiés statutaires et solliciteurs d'asile »!

11. -Catégorie inventée par quelques sociologues (Altan Gökalp, Nadine Weibel) pour qualifier le commerce par les Turcs de produits réputés licites selon la Sunna et donc halal, helâl en turc : viandes, charcuteries, produits alimentaires, vite rejoints par d'autres produits (vêtements tesettür) ou services commercialisés par des groupes mettant en avant leur conviction religieuse à fin toute commerciale.

12. -Il n'est pas rare que, venus en touristes et sans visas, des familles albanaises, kosovares ou bosniaques, s'installent chez des proches, familles ou amis ; leur présence peut être relevée dans certains quartiers des grandes agglomérations par le stationnement des voitures qui ont gardé leur plaque d'immatriculation originale. Le quartier d'Istanbul nommé Yenibosna (Nouvelle Bosnie !) attire ainsi de nombreux Bosniaques et Albanais.

13. -Entretien avec M. l'Attaché de Police près l'Ambassade de France à Ankara le 12.11.1999, entretien téléphonique et échanges de fax avec le Directeur des Relations Internationales à la Direction générale de la Sécurité, Ankara, dans le cadre de la mission IHESI, projet ODYSSEUS. Rencontre à Bruxelles avec le Président de la Direction des Etrangers et des Frontières.

14. -Paul Henze travaille à la Rand Corporation (USA) et Enders Wimbush a été responsable de Radio Liberty à Munich. Le journaliste de Cumhuriyet Ugur Mumcu s'est souvent opposé à Paul Henze qu'il décrit comme étant le responsable de la CIA pour la Turquie.

15. -La physionomie très turque de l'auteur de ces lignes lui aura valu de nombreuses fois de changer des francs français au taux ouvrier et de fréquenter indifféremment banques et officines de change sans jamais être qualifié de touriste. Les banques demandent encore, pas toujours, un passeport, les bureaux de change jamais. Les statistiques doivent nécessairement s'en ressentir ! La multiplication des guichets automatiques devrait faciliter la production statistique sur l'origine des devises. Sinon, n'importe quelle station-service, n'importe quel chauffeur de taxi ou commerçant, se fera un plaisir de jouer les agents de change.

16. -Entretien le 24 novembre 1999 avec le Directeur Général Adjoint du service lors de la mission IHESI - projet ODYSSEUS à Ankara.

17. -Le relais peut être ici pris par la consultation des Lloyd's Register of Shipping - un bureau est ouvert au public à Paris - ou le réseau Internet pour les compagnies 
maritimes grecques, dont certaines, à l'image de Minoan Lines, font un remarquable travail de communication.

18. -Pour ne citer que deux exemples, la collection éditée par la Chambre de Commerce d'Istanbul, une quarantaine de titres sur les pays partenaires de la Turquie, à la fois guides pratiques et études économiques, parfois fondés sur des enquêtes solides auprès des expatriés (Autriche, Pologne, ...) ou les publications sur Internet du DEIK (Kazakhstan).

19. -On peut citer nombre d'enquêtes du journaliste d'investigation turc, Ugur Mumcu, sur les transferts de fonds entre la Turquie et divers pays d'Europe, dont évidemment la Suisse. Ces très nombreuses enquêtes sur les groupes politiques, réseaux de délinquance, terroristes, clandestins divers, forment une partie importante du travail d'un journaliste connu pour son indépendance d'esprit et victime d'un attentat devant son domicile d'Ankara le 24 janvier 1993. Parmi les titres disponibles aujourd'hui - une maison d'édition a été créée et dédiée à sa mémoire et à celle des journalistes victimes du terrorisme - on relève : Trafic d'armes et terreur ; Le Pape, la Mafia et Agca ; Rabita (organisation saoudienne) ; Confrérie, Politique et Commerce ; Nationalisme S.A. ; Droit, Etat et Tribu ; ...

\section{AUTEUR}

\section{STÉPHANE DE TAPIA}

Chargé de Recherche CNRS, CeRATO - CNRS 23, rue du Lœss, Bât. 50 F-67037 Strasbourg Cedex 02, France, associé à MIGRINTER, UMR 7043, téléphone : (33) 3.88.10.60.90.

stephane.detapia@c-strasbourg.fr 